\title{
QUASI-STATIC RATE-INDEPENDENT EVOLUTIONS: CHARACTERIZATION, EXISTENCE, APPROXIMATION AND APPLICATION TO FRACTURE MECHANICS *
}

\author{
MATteo NeGRI ${ }^{1}$
}

\begin{abstract}
We characterize quasi-static rate-independent evolutions, by means of their graph parametrization, in terms of a couple of equations: the first gives stationarity while the second provides the energy balance. An abstract existence result is given for functionals $\mathcal{F}$ of class $C^{1}$ in reflexive separable Banach spaces. We provide a couple of constructive proofs of existence which share common features with the theory of minimizing movements for gradient flows. Moreover, considering a sequence of functionals $\mathcal{F}_{n}$ and its $\Gamma$-limit $\mathcal{F}$ we provide, under suitable assumptions, a convergence result for the associated quasi-static evolutions. Finally, we apply this approach to a phase field model in brittle fracture.
\end{abstract}

Mathematics Subject Classification. 49J27, 74R10, 58E30.

Received May 8, 2013.

Published online August 4, 2014.

\section{INTRODUCTION}

In the last few years the analysis of quasi-static rate-independent evolutions has been the object of several important advances. Theoretical and applied results have been developed essentially along two alternative lines: on the one hand the evolutions by global minima (usually named "energetic evolutions") on the other the evolutions by critical points.

To illustrate the picture in a simple setting, consider a stored energy $\mathcal{E}:\left[t_{0}, t_{1}\right] \times \mathcal{V} \rightarrow \mathbb{R}$, where $\left[t_{0}, t_{1}\right]$ is the time interval and $\mathcal{V}$ is a separable reflexive Banach space (either finite or infinite dimensional) together with a dissipation distance $\Delta: \mathcal{V} \times \mathcal{V} \rightarrow[0,+\infty]$. Given an initial condition $v\left(t_{0}\right)=v_{0}$ (with $v_{0}$ globally stable) a trajectory $v:\left[t_{0}, t_{1}\right] \rightarrow \mathcal{V}$ is an energetic evolution [24] if the following conditions hold,

$(S)$ for every $t \in\left[t_{0}, t_{1}\right]$ it holds

$$
\mathcal{E}(t, v(t)) \leq \mathcal{E}(t, \phi)+\Delta(\phi, v(t)) \quad \text { for every } \phi \in \mathcal{V}
$$

\footnotetext{
Keywords and phrases. Quasi-static evolutions, phase-field.

* This material is based on work supported by ERC under Grant No. 290888 "Quasistatic and Dynamic Evolution Problems in Plasticity and Fracture", by GNAMPA under project "Modelli variazionali per la propagazione di fratture, la delaminazione e il danneggiamento" and by MIUR under project "Calculus of Variations".

1 Department of Mathematics, University of Pavia, Via A. Ferrata 1, 27100 Pavia, Italy. matteo.negri@unipv.it
} 
(E) for every $t \in\left[t_{0}, t_{1}\right]$ it holds

$$
\mathcal{E}(t, v(t))=\mathcal{E}\left(t_{0}, v\left(t_{0}\right)\right)-\Delta\left(v(t), v\left(t_{0}\right)\right)+\int_{t_{0}}^{t} \partial_{t} \mathcal{E}(s, v(s)) \mathrm{d} s .
$$

The letters $(S)$ and $(E)$ denote respectively (global) stability and energy balance. Assuming further that there exists a dissipation "potential" $\mathcal{D}: \mathcal{V} \rightarrow[0,+\infty)$ such that $\Delta(v, w)=\mathcal{D}(v)-\mathcal{D}(w)$ and introducing the energy functional $\mathcal{F}(t, v)=\mathcal{E}(t, v)+\mathcal{D}(v)$ the $(S)-(E)$ conditions read

$(S)$ for every $t \in\left[t_{0}, t_{1}\right]$ it holds

$$
\mathcal{F}(t, v(t)) \leq \mathcal{F}(t, \phi) \quad \text { for every } \phi \in \mathcal{V}
$$

(E) for every $t \in\left[t_{0}, t_{1}\right]$ it holds

$$
\mathcal{F}(t, v(t))=\mathcal{F}\left(t_{0}, v\left(t_{0}\right)\right)+\int_{t_{0}}^{t} \partial_{t} \mathcal{F}(s, v(s)) \mathrm{d} s .
$$

Often a representation of the form $\Delta(v, w)=\mathcal{D}(v)-\mathcal{D}(w)$ holds under some constraint on the admissible increment (it is indeed the case in brittle and cohesive fracture and in associative plasticity), however, to keep the presentation clear, we will not introduce any constraint in the abstract picture; we will see how to deal with a constrained problem in Section 8. It is important to highlight that in the above definition there are no derivatives of the stored energy $\mathcal{E}$ with respect to the state variable $v$, thanks to this fact it is possible to prove existence of energetic evolutions under (very) low regularity assumptions on $\mathcal{E}$, including the case of spaces without a vectorial structure, see for instance [13] for an application to fracture. On the other hand, it may happen that the behaviour of energetic solutions is not physically admissible: when a discontinuity occurs the evolution typically "tunnels" under an energy barrier.

Let us turn to evolutions by critical points, assuming from now on that $\mathcal{F}$ is of class $C^{1}$. In the literature there are several equivalent definitions, most of them formulated in terms of the trajectory $t \mapsto v(t)$, as the $B V$ solutions of $[26,27]$. Here we will adopt a graph parametrization of solutions, inspired by [16], which provides in turn a parametrized $B V$ solution (see Sect. 3 and [25]). The idea is to define the evolution by means of a (Lipschitz) parametrization of the extended graph, of the type $\tau \mapsto(t(\tau), v(\tau))$ for $\tau \in[0, \mathcal{T})$. This is a convenient choice to focus on discontinuities, indeed with this parametrization jumps at a certain time $t_{s}$ are represented by "vertical parts" of the extended graph, of the form $\tau \mapsto(t(\tau), v(\tau))$ for $\tau \in\left[\tau^{-}, \tau^{+}\right]$with $t(\tau)=t_{s}$ and $v\left(\tau^{-}\right) \neq v\left(\tau^{+}\right)$. Adopting graph parametrization, our goal is first to provide a definition which resembles the $(S)-(E)$ formulation above and then to provide an existence result in separable reflexive Banach spaces. Let us see in more detail our definition: let $\tau \mapsto(t(\tau), v(\tau))$ be a Lipschitz map with $0 \leq t^{\prime} \leq 1$ and $\left\|v^{\prime}\right\| \leq 1\left(t^{\prime}\right.$ and $v^{\prime}$ denote the derivatives with respect to $\tau$ ). We will say that $(t, v)$ is (a parametrization of) a quasi-static evolution if

$\left(S^{\prime}\right)$ for every $\tau$ with $t^{\prime}(\tau)>0$ it holds

$$
\left\|\partial_{v} \mathcal{F}(t(\tau), v(\tau))\right\|=0
$$

$\left(E^{\prime}\right)$ for every $\tau$ it holds

$$
\mathcal{F}(t(\tau), v(\tau))=\mathcal{F}(t(0), v(0))-\int_{0}^{\tau}\left\|\partial_{v} \mathcal{F}(t(s), v(s))\right\| \mathrm{d} s+\int_{0}^{\tau} \partial_{t} \mathcal{F}(t(s), v(s)) t^{\prime}(s) \mathrm{d} s,
$$

where $\left\|\partial_{v} \mathcal{F}(t, v)\right\|$ is the norm in the dual $\mathcal{V}^{\prime}$, i.e.

$$
\left\|\partial_{v} \mathcal{F}(t, v)\right\|=\max \left\{\partial_{v} \mathcal{F}(t, v)[\phi]:\|\phi\| \leq 1\right\} .
$$

Here the labels $\left(S^{\prime}\right)$ and $\left(E^{\prime}\right)$ stand respectively for stationarity and energy balance while the prime symbol suggests the dependence on derivatives of the stored energy. Close to our definition of evolutions by critical 
points are those of $[16,26,32,37]$ while close to our existence proof are those on minimizing movements for gradient flows, e.g. $[1,2,15,33,35]$.

Several properties of the evolution follow from this definition. First of all, note that $\left(S^{\prime}\right)$ can be written also in the (norm free) form

$$
\partial_{v} \mathcal{F}(t(\tau), v(\tau))[\phi]=0 \quad \text { for every } \phi \in \mathcal{V} .
$$

Next, if $t^{\prime}(s)>0$ for every $s \in\left[\tau_{1}, \tau_{2}\right]$ (and thus there are no jump discontinuities in $\left[t\left(\tau_{1}\right), t\left(\tau_{2}\right)\right]$ ) then $\left(E^{\prime}\right)$ yields

$$
\mathcal{F}\left(t\left(\tau_{2}\right), v\left(\tau_{2}\right)\right)=\mathcal{F}\left(t\left(\tau_{1}\right), v\left(\tau_{1}\right)\right)+\int_{\tau_{1}}^{\tau_{2}} \partial_{t} \mathcal{F}(t(s), v(s)) t^{\prime}(s) \mathrm{d} s
$$

which, up to a change of variable, is equivalent to $(E)$. On the contrary, if $t^{\prime}(s)=0$ for every $s \in\left[\tau^{-}, \tau^{+}\right]$(and thus there is a jump discontinuity at time $t=t(s))$ then $\left(E^{\prime}\right)$ reads

$$
\mathcal{F}\left(t, v\left(\tau^{+}\right)\right)=\mathcal{F}\left(t, v\left(\tau^{-}\right)\right)-\int_{\tau^{-}}^{\tau^{+}}\left\|\partial_{v} \mathcal{F}(t, v(s))\right\| \mathrm{d} s,
$$

and in particular $\mathcal{F}\left(t, v\left(\tau_{2}\right)\right) \leq \mathcal{F}\left(t, v\left(\tau_{1}\right)\right)$ for every $\tau^{-} \leq \tau_{1}<\tau_{2} \leq \tau^{+}$. Most important, the path $\tau \mapsto v(\tau)$ between $v\left(\tau^{-}\right)$and $v\left(\tau^{+}\right)$is a curve of maximal (normalized) slope [2] for the autonomous functional $\mathcal{F}(t, \cdot)$; this property will follow from the optimality of $v^{\prime}(\tau)$ described hereafter. By the chain rule we can write

$$
d_{\tau} \mathcal{F}(t(\tau), v(\tau))=\partial_{v} \mathcal{F}(t(\tau), v(\tau))\left[v^{\prime}(\tau)\right]+\partial_{t} \mathcal{F}(t(\tau), v(\tau)) t^{\prime}(\tau)
$$

and thus for $\tau_{1}<\tau_{2}$

$$
\begin{aligned}
\mathcal{F}\left(t\left(\tau_{2}\right), v\left(\tau_{2}\right)\right) & =\mathcal{F}\left(t\left(\tau_{1}\right), v\left(\tau_{1}\right)\right)+\int_{\tau_{1}}^{\tau_{2}} d_{\tau} \mathcal{F}(t(\tau), v(\tau)) \mathrm{d} s \\
& =\mathcal{F}\left(t\left(\tau_{1}\right), v\left(\tau_{1}\right)\right)+\int_{\tau_{1}}^{\tau_{2}} \partial_{v} \mathcal{F}(t(\tau), v(\tau))\left[v^{\prime}(\tau)\right] \mathrm{d} \tau+\int_{\tau_{1}}^{\tau_{2}} \partial_{t} \mathcal{F}(t(\tau), v(\tau)) t^{\prime}(\tau) \mathrm{d} \tau
\end{aligned}
$$

On the other hand, by $\left(E^{\prime}\right)$ we can write

$$
\mathcal{F}\left(t\left(\tau_{2}\right), v\left(\tau_{2}\right)\right)=\mathcal{F}\left(t\left(\tau_{1}\right), v\left(\tau_{1}\right)\right)-\int_{\tau_{1}}^{\tau_{2}}\left\|\partial_{v} \mathcal{F}(t(\tau), v(\tau))\right\| \mathrm{d} \tau+\int_{\tau_{1}}^{\tau_{2}} \partial_{t} \mathcal{F}(t(\tau), v(\tau)) t^{\prime}(\tau) \mathrm{d} \tau
$$

Therefore, $\partial_{v} \mathcal{F}(t(\tau), v(\tau))\left[v^{\prime}(\tau)\right]=-\left\|\partial_{v} \mathcal{F}(t(\tau), v(\tau))\right\|$ for a.e. $\tau$. Since $\left\|v^{\prime}\right\| \leq 1$ it follows that for a.e. $\tau$

$$
v^{\prime}(\tau) \in \operatorname{argmin}\left\{\partial_{v} \mathcal{F}(t(\tau), v(\tau))[\phi]:\|\phi\| \leq 1\right\} .
$$

If $t^{\prime}(\tau)>0$ the above property is not of interest since $\partial_{v} \mathcal{F}(t(\tau), v(\tau))=0$ and thus any $v^{\prime}$ is a minimizer. On the contrary, on jump discontinuities, where $\partial_{v} \mathcal{F}(t(\tau), v(\tau)) \neq 0$, it says that $v^{\prime}(\tau)$ is the steepest descent direction; in a Hilbert space setting, $v$ would solve the normalized gradient flow

$$
v^{\prime}(\tau)=-\nabla \mathcal{F}(t, v(\tau)) /\|\nabla \mathcal{F}(t, v(\tau))\|,
$$

where $\nabla \mathcal{F}$ is the gradient of $\mathcal{F}$ (with respect to the norm $\|\cdot\|$ ), $\tau$ belongs to the jump interval $\left[\tau^{-}, \tau^{+}\right]$and $t=t(\tau)$ is the discontinuity point. To understand the idea behind the normalization of the gradient consider at time $t$ a transition between the equilibrium configurations $v\left(t^{-}\right)$and $v\left(t^{+}\right)$. Since $v\left(t^{-}\right)$and $v\left(t^{+}\right)$are equilibrium configurations a (non-normalized) gradient flow would provide in general a transition in an infinite interval, say $\left[\tau^{-},+\infty\right)$, and thus it would not be possible to extend the parametrization after the time $t$. On the contrary, a normalized gradient flow allows to find a parametrization of the evolution in a finite interval, say $\left[\tau^{-}, \tau^{+}\right]$, whenever the length of the path $\tau \mapsto v(\tau)$ is finite. 
Under suitable conditions on the energy functional, we prove existence of such an evolution both by means of a "parametrized" minimizing movement and by means of a forward Euler scheme; the proofs, based on sequences of incremental problems, do not employ vanishing viscosity arguments. The common structure of the existence results could be summarized as: construction of a discrete evolution, discrete stationarity and discrete energy inequality, construction of a continuum interpolation, compactness, convergence of stationarity and energy inequality to their continuum counterparts $\left(E^{\prime}\right)$ and $\left(S^{\prime}\right)$.

Next, following the scheme of [34], we provide an approximation result of the following type. Consider a sequence of functionals $\mathcal{F}_{n}$ together with the corresponding quasi-static evolutions, say $\left(t_{n}, v_{n}\right)$. Assume that $\mathcal{F}_{n} \Gamma$-converge to $\mathcal{F}$ with respect to convergence in $t$ and weak convergence in $v$. Under suitable conditions on the convergence of $\left\|\partial_{v} \mathcal{F}_{n}\right\|$ and $\partial_{t} \mathcal{F}_{n}$ to $\left\|\partial_{v} \mathcal{F}\right\|$ and $\partial_{t} \mathcal{F}$ respectively, we show that the quasi-static evolutions $\left(t_{n}, v_{n}\right)$ converge (up to subsequences) to a quasi-static evolution $(t, v)$ for the $\Gamma$-limit $\mathcal{F}$. The proof is based on the same arguments developed for the convergence of discrete stationarity and energy inequality, which appear in the existence result. We could consider this result as the analogue of [29], developed for energetic evolutions.

Moreover, we consider the quasi-static evolution with respect to a weaker norm, induced by the continuous immersion of $\mathcal{V}$ in a reflexive separable Banach space $\mathcal{W}$. In this context the existence result developed in the norm of $\mathcal{V}$ is not suitable, we follow instead a "Galerkin approach" approximating the evolution in finite dimensional spaces and then passing to the limit by virtue of the approximation result.

The last part of the paper is dedicated to quasi-static evolutions for a phase field model in brittle fracture [6]. The presence of a constraint, related to the irreversibility of the crack, does not change the core of the previous arguments; indeed we prove existence of a parametrized quasi-static evolution in the $H^{1}$ and $L^{2}$ norm. The reader interested in phase field models for fracture will find also an energetic evolution in [19], an $L^{2}$-gradient flow (in the displacement) in [4], an $L^{1}$-viscosity solution in [21] and a dynamic visco-elastic evolution in [23]. Besides our interest for the specific application, this example shows that the formulation and the existence result, both with some modifications, are suitable also in the case of constrained problems.

\section{Definitions And statements of the main Results}

Let $\mathcal{V}$ be a reflexive, separable Banach space, with norm $\|\cdot\|$, and let $\left[t_{0}, t_{1}\right]$ be a time interval. Let $\mathcal{F}$ : $\left[t_{0}, t_{1}\right] \times \mathcal{V} \rightarrow[0,+\infty)$ be an energy functional. Assume that $\mathcal{F}$ is of class $C^{1}$. Within this setting the quasi-static evolutions of interest in this work are characterized by the following definition.

Definition 2.1. Let $(t, v):[0, \mathcal{T}) \rightarrow\left[t_{0}, t_{1}\right] \times \mathcal{V}$ be a Lipschitz map with $(t(0), v(0))=\left(t_{0}, v_{0}\right), 0 \leq t^{\prime} \leq 1$ and $\left\|v^{\prime}\right\| \leq 1 ;(t, v)$ is (a parametrization of) a quasi-static evolution if

$\left(S^{\prime}\right)$ the following stationarity condition holds: for every $\tau$ with $t^{\prime}(\tau)>0$

$$
\left\|\partial_{v} \mathcal{F}(t(\tau), v(\tau))\right\|=0,
$$

$\left(E^{\prime}\right)$ the following energy balance holds: for every $\tau$

$$
\mathcal{F}(t(\tau), v(\tau))=\mathcal{F}\left(t_{0}, v_{0}\right)-\int_{0}^{\tau}\left\|\partial_{v} \mathcal{F}(t(s), v(s))\right\| \mathrm{d} s+\int_{0}^{\tau} \partial_{t} \mathcal{F}(t(s), v(s)) t^{\prime}(s) \mathrm{d} s,
$$

where $\left\|\partial_{v} \mathcal{F}(t, v)\right\|$ denotes the norm in the dual space $\mathcal{V}^{\prime}$.

In general, according to the previous Definition, if $v_{0}$ is an equilibrium configuration the trivial parametrization $\tau \mapsto\left(t_{0}, v_{0}\right)$ is an admissible quasi-static evolution. Note also that using parametrizations it may happen that the solution is instead a pure jump, of the form $\left(t_{0}, v(\tau)\right)$, which is the only possible solution when there are no equilibrium configurations. To conclude, note that the continuity of $\partial_{t} \mathcal{F}$ and $\partial_{v} \mathcal{F}$ are enough for integrability in (2.2) for every Lipschitz parametrization.

In the next two sections we will prove the following existence result by means of both a minimizing movement and an explicit Euler scheme. 
Theorem 2.2. Let $\mathcal{F}:\left[t_{0}, t_{1}\right] \times \mathcal{V} \rightarrow[0,+\infty)$ be of class $C^{1}$ with

$$
\begin{gathered}
\mathcal{F}(t, v) \leq \liminf _{m} \mathcal{F}\left(t_{m}, v_{m}\right) \quad \text { for } t_{m} \rightarrow t \text { and } v_{m} \rightarrow v, \\
\left\|\partial_{v} \mathcal{F}(t, v)\right\| \leq \liminf _{m}\left\|\partial_{v} \mathcal{F}\left(t_{m}, v_{m}\right)\right\| \quad \text { for } t_{m} \rightarrow t \text { and } v_{m} \rightarrow v, \\
\partial_{t} \mathcal{F}(t, v)=\lim _{m} \partial_{t} \mathcal{F}\left(t_{m}, v_{m}\right) \quad \text { for } t_{m} \rightarrow t \text { and } v_{m} \rightarrow v .
\end{gathered}
$$

Moreover assume that there exists a non-decreasing modulus of continuity $\mathcal{C}$ such that

$$
\left\|\partial_{v} \mathcal{F}(t, v)-\partial_{v} \mathcal{F}(t, w)\right\|+\left|\partial_{t} \mathcal{F}(t, v)-\partial_{t} \mathcal{F}(t, w)\right| \leq \mathcal{C}(\|v-w\|) \quad \text { uniformly w.r.t. } t \in\left[t_{0}, t_{1}\right] .
$$

Under the above hypotheses there exists a quasi-static evolution in the sense of Definition 2.1.

Note that the coercivity of the functional $\mathcal{F}(t, \cdot)$ is not required for existence. Note also that in several cases, usually in the presence of irreversibility constraints, it is possible (see Lem. 3.2 in [16], and Sect. 8) to guarantee the existence of non-trivial solutions.

The next Theorem, in the spirit of $[8,34,35]$, shows the connection between $\Gamma$-convergence $[7,11]$ of energy functionals and the associated quasi-static evolutions (the proof is contained in Sect. 6). A typical application of this Theorem is to finite element approximation, however it can be used also as an existence result (as we will see in the sequel).

Theorem 2.3. Let $\mathcal{F}_{h}:\left[t_{0}, t_{1}\right] \times \mathcal{V}_{h} \rightarrow[0,+\infty)$ be a family of $C^{1}$ functionals defined on the subspaces $\mathcal{V}_{h} \subset \mathcal{V}$. Assume that $\mathcal{V}_{h}$, endowed with the norm of $\mathcal{V}$, are Banach spaces. Let $\left(t_{h}, v_{h}\right):[0, \mathcal{T}) \rightarrow\left[t_{0}, t_{1}\right] \times \mathcal{V}_{h}$ be a family of quasi-static evolutions for $\mathcal{F}_{h}$ (in the sense of Def. 2.1) with initial condition $\left(t_{0}, v_{h, 0}\right)$. Let $\mathcal{F}:\left[t_{0}, t_{1}\right] \times \mathcal{V} \rightarrow$ $[0,+\infty)$ of class $C^{1}$. Assume that

$$
\begin{aligned}
\mathcal{F}(t, v) & \leq \liminf _{h} \mathcal{F}_{h}\left(t_{h}, v_{h}\right) \quad \text { for } t_{h} \rightarrow t \text { and } v_{h} \rightarrow v \text { in } \mathcal{V}, \\
\left\|\partial_{v} \mathcal{F}(t, v)\right\| & \leq \liminf _{h}\left\|\partial_{v} \mathcal{F}_{h}\left(t_{h}, v_{h}\right)\right\|_{h} \quad \text { for } t_{h} \rightarrow t \text { and } v_{h} \rightarrow v \text { in } \mathcal{V}, \\
\partial_{t} \mathcal{F}(t, v) & =\lim _{h} \partial_{t} \mathcal{F}_{h}\left(t_{h}, v_{h}\right) \quad \text { for } t_{h} \rightarrow t \text { and } v_{h} \rightarrow v \text { in } \mathcal{V},
\end{aligned}
$$

where $\left\|\partial_{v} \mathcal{F}_{h}\left(t_{h}, v_{h}\right)\right\|_{h}$ is the norm in the dual $\mathcal{V}_{h}^{\prime}$. Assume also that the initial condition is "well-prepared", i.e. that

$$
v_{h, 0} \rightarrow v_{0} \text { in } \mathcal{V} \text { and that } \mathcal{F}_{h}\left(t_{0}, v_{h, 0}\right) \rightarrow \mathcal{F}\left(t_{0}, v_{0}\right),
$$

and that the power $\partial_{t} \mathcal{F}_{h}\left(t_{h}(s), v_{h}(s)\right)$ is uniformly bounded. Then there exists a subsequence (not relabelled) such that $t_{h}(\tau) \rightarrow t(\tau)$ and $v_{h}(\tau) \rightarrow v(\tau)$, for every $\tau \in[0, \mathcal{T})$; the limit $(t, v)$ is a quasi-static evolution for $\mathcal{F}$ (in the sense of Def. 2.1) with initial condition $\left(t_{0}, v_{0}\right)$.

Finally, consider the Banach space $\mathcal{V}$ to be continuously embedded in a Banach space $\mathcal{W}$ and endowed with the norm of $\mathcal{W}$. (The prototype example is the inclusion of $H^{1}$ in $L^{2}$ ). Let $\mathcal{F}:\left[t_{0}, t_{1}\right] \times \mathcal{V} \rightarrow[0, \infty)$ be again of class $C^{1}$. Clearly $\partial_{v} \mathcal{F}(t, v)[\cdot]$ is linear and continuous on $\mathcal{V}$, endowed with the norm $\|\cdot\|_{\mathcal{V}}$. If $\partial_{v} \mathcal{F}(t, v)[\cdot]$ is also linear and continuous on $\mathcal{V}$, endowed with the norm $\|\cdot\|_{\mathcal{W}}$, it is possible to define also

$$
\left\|\nabla_{\mathcal{W}} \mathcal{F}(t, v)\right\|=\sup \left\{\partial_{v} \mathcal{F}(t, v)[\phi]: \phi \in \mathcal{V},\|\phi\|_{\mathcal{W}} \leq 1\right\} .
$$

If $\partial_{v} \mathcal{F}(t, v)[\cdot]$ is not linear and continuous on $\mathcal{V}$, endowed with the norm $\|\cdot\|_{\mathcal{W}}$, we set $\left\|\nabla_{\mathcal{W}} \mathcal{F}(t, v)\right\|=\infty$. Within this framework we can define the quasi-static evolution with respect to the norm $\|\cdot\|_{\mathcal{W}}$ exactly as we did in Definition 2.1. 
Definition 2.4. Let $(t, v):[0, \mathcal{T}) \rightarrow\left[t_{0}, t_{1}\right] \times \mathcal{V}$ be a Lipschitz map with $(t(0), v(0))=\left(t_{0}, v_{0}\right), 0 \leq t^{\prime} \leq 1$ and $\left\|v^{\prime}\right\|_{\mathcal{W}} \leq 1 ;(t, v)$ is (a parametrization of) a quasi-static evolution if

$\left(S^{\prime}\right)$ for every $\tau$ with $t^{\prime}(\tau)>0$

$$
\left\|\nabla_{\mathcal{W}} \mathcal{F}(t(\tau), v(\tau))\right\|=0
$$

$\left(E^{\prime}\right)$ for every $\tau$

$$
\mathcal{F}(t(\tau), v(\tau))=\mathcal{F}\left(t_{0}, v_{0}\right)-\int_{0}^{\tau}\left\|\nabla_{\mathcal{W}} \mathcal{F}(t(s), v(s))\right\| \mathrm{d} s+\int_{0}^{\tau} \partial_{t} \mathcal{F}(t(s), v(s)) t^{\prime}(s) \mathrm{d} s .
$$

In the weak norm setting the existence result provided by Theorem 2.2 is not really useful since the uniform continuity of the derivatives, with respect to $\|\cdot\|_{\mathcal{W}}$, is often too restrictive (think of the Dirichlet energy with respect to $L^{2}$ ). However, using a sequence of finite dimensional approximations together with Theorem 2.3 we will prove in Section 7 the following existence result.

Theorem 2.5. Let $\mathcal{F}:\left[t_{0}, t_{1}\right] \times \mathcal{V} \rightarrow[0,+\infty)$ of class $C^{1}$ and such that

$$
\begin{array}{rlrl}
\mathcal{F}(t, v) & \leq \liminf _{m} \mathcal{F}\left(t_{m}, v_{m}\right) \quad & \text { for } t_{m} \rightarrow t \text { and } v_{m} & \rightarrow v \text { in } \mathcal{V}, \\
\partial_{v} \mathcal{F}(t, v)[\phi] & =\lim _{m} \partial_{v} \mathcal{F}\left(t_{m}, v_{m}\right)[\phi] \quad \text { for } t_{m} \rightarrow t, v_{m} \rightarrow v \text { in } \mathcal{V} \text { and } \phi \in \mathcal{V}, \\
\partial_{t} \mathcal{F}(t, v) & =\lim _{m} \partial_{t} \mathcal{F}\left(t_{m}, v_{m}\right) \quad \text { for } t_{m} \rightarrow t \text { and } v_{m} \rightarrow v \text { in } \mathcal{V} .
\end{array}
$$

Moreover assume that there exists a non-decreasing modulus of continuity $\mathcal{C}$ s.t.

$$
\left\|\partial_{v} \mathcal{F}(t, v)-\partial_{v} \mathcal{F}(t, w)\right\|+\left|\partial_{t} \mathcal{F}(t, v)-\partial_{t} \mathcal{F}(t, w)\right| \leq \mathcal{C}(\|v-w\|) \quad \text { uniformly w.r.t. } t \in\left[t_{0}, t_{1}\right] .
$$

Let $\mathcal{V}$ be continuously embedded in a separable reflexive Banach space $\mathcal{W}$. Assume also that $\mathcal{F}$ is coercive with respect to $\|\cdot\|_{\mathcal{V}}$ and that for some $A, B$ independent of $t$ and $v$ it holds

$$
\left|\partial_{t} \mathcal{F}(t, v)\right| \leq A \mathcal{F}(t, v)+B .
$$

Then there exists a quasi-static evolution in the sense of Definition 2.4.

Before proving the above results, it is fair to mention that the statements are far from being sharp, for instance, (2.5) could be replaced by a limsup condition, the uniform continuity of (2.6) could be made time dependent while conditions (2.7)-(2.9) could include the energy excess, as in [34]. In this work our goal is indeed to present a scheme and the essential ingredients, rather than providing a general framework.

\section{COMParison With $B V$ SOlutions}

We will start presenting an abstract framework for $B V$ solutions, making reference to $[25,28]$ for the general and precise assumptions. Then we will see how our parametrized solutions fits into the above framework.

Let $\mathcal{V}$ be a reflexive, separable Banach space. Denote by $\mathcal{E}:\left[t_{0}, t_{1}\right] \times \mathcal{V} \rightarrow \mathbb{R}$ the stored energy and by $\mathcal{R}: \mathcal{V} \times \mathcal{V} \rightarrow[0,+\infty]$ the dissipation functional. We will write $\mathcal{E}(t, v)$ and $\mathcal{R}(v, z)$ having in mind that $v$ denotes the state variable while $z$ denotes the velocity, say $\dot{v}$. Note that in $[25,28]$ the dissipation is actually of the form $\mathcal{R}(z)$. With this notation the dissipation distance between the states $v_{0}$ and $v_{1}$ is

$$
\operatorname{Dist}_{\mathcal{R}}\left(v_{0}, v_{1}\right)=\inf \left\{\int_{0}^{1} \mathcal{R}\left(v(s), v^{\prime}(s)\right) \mathrm{d} s: v \in W^{1,1}([0,1], \mathcal{V}), v(0)=v_{0}, v(1)=v_{1}\right\} .
$$

For $B V$ trajectories (in analogy with the variation) the amount of dissipated energy is given by

$$
\operatorname{Diss}_{B V}\left(v,\left[t_{a}, t_{b}\right]\right)=\sup \left\{\sum_{i=0}^{m} \operatorname{Dist}_{\mathcal{R}}\left(v_{i}, v_{i+1}\right): v_{i}=v\left(t_{i}\right), t_{a} \leq t_{0}<\ldots<t_{m+1}=t_{b}\right\} .
$$


Now, consider the $\varepsilon$-viscous dissipation functional $\mathcal{R}_{\varepsilon}(v, z)=\mathcal{R}(v, z)+(\varepsilon / 2)\|z\|^{2}$ together with its Legendre transform $\mathcal{R}_{\varepsilon}^{*}(v, \xi)$. We then introduce the vanishing viscosity contact potential

$$
\mathfrak{p}(v, z, \xi) \inf _{\varepsilon>0}\left\{\mathcal{R}_{\varepsilon}(v, z)+\mathcal{R}_{\varepsilon}^{*}(v, \xi)\right\}
$$

and finally the Finsler distance

$$
\operatorname{Dist}_{\mathfrak{p}}\left(t, v_{0}, v_{1}\right)=\inf \left\{\int_{0}^{1} \mathfrak{p}\left(v(s), v^{\prime}(s),-\partial_{v} \mathcal{E}(t, v(s))\right) \mathrm{d} s: v \in W^{1,1}([0,1], \mathcal{V}), v(0)=v_{0}, v(1)=v_{1}\right\} .
$$

We have now all the ingredients to define a $B V$ solution, for sake of simplicity we will restrict ourselves to the case of left continuous solutions for energies of class $C^{1}$. We denote by $J(v)$ the set of jump points of $v$. A (left-continuous) map $v \in B V\left(\left[t_{0}, t_{1}\right], \mathcal{V}\right)$ with $v\left(t_{0}\right)=v_{0}$ is a $B V$ solution if

$(\bar{S})$ for $t \in\left[t_{0}, t_{1}\right] \backslash J(v)$ it holds

$$
\partial_{z} \mathcal{R}(v(t), 0)+\partial_{v} \mathcal{E}(t, v(t)) \ni 0,
$$

$(\bar{E})$ for every $t$ it holds

$$
\begin{array}{r}
\mathcal{E}(t, v(t))+\operatorname{Diss}_{B V}\left(v,\left[t_{0}, t\right]\right)+\sum_{s \in J(v) \cap\left[t_{0}, t\right)} \operatorname{Dist}_{\mathfrak{p}}\left(s, v(s), v\left(s^{+}\right)\right)-\operatorname{Dist}_{\mathcal{R}}\left(v(s), v\left(s^{+}\right)\right) \\
=\mathcal{E}\left(t_{0}, v_{0}\right)+\int_{t_{0}}^{t} \partial_{t} \mathcal{E}(s, v(s)) \mathrm{d} s .
\end{array}
$$

Now let us re-write the above conditions remembering that in our setting the dissipation functional $\mathcal{R}$ admits a potential $\mathcal{D}: \mathcal{V} \rightarrow[0,+\infty)$ (depending only on the state variable $v$ ) such that $\mathcal{R}(v, z)=\partial_{v} \mathcal{D}(v)[z]$. Therefore we have

$$
\partial_{z} \mathcal{R}(v, z)[\phi]=\partial_{v} \mathcal{D}(v)[\phi] \quad \text { for every } \phi \in \mathcal{V} .
$$

In particular $\partial_{z} \mathcal{R}(v, 0)=\partial_{v} \mathcal{D}(v)$ and then $(\bar{S})$ reads: for $t \in\left[t_{0}, t_{1}\right] \backslash J(v)$ it holds

$$
\partial_{v} \mathcal{D}(v(t))+\partial_{v} \mathcal{E}(t, v(t))=0 .
$$

In terms of the free energy $\mathcal{F}(t, v)=\mathcal{E}(t, v)+\mathcal{D}(v)$ the above equation becomes the Euler-Lagrange equation $\partial_{v} \mathcal{F}(t, v(t))=0$. Moreover, if $\mathcal{D}$ is of class $C^{1}$ and $v$ left-continuous the equilibrium equation $\partial_{v} \mathcal{F}(t, v(t))=0$ holds for every $t \in\left(t_{0}, t\right]$.

Next, for an absolutely continuous map $s \mapsto v(s)$ we have

$$
d_{s} \mathcal{D}(v(s))=\partial_{v} \mathcal{D}(v(s))\left[v^{\prime}(s)\right]=\mathcal{R}\left(v(s), v^{\prime}(s)\right)
$$

and thus

$$
\int_{0}^{1} \mathcal{R}\left(v(s), v^{\prime}(s)\right) \mathrm{d} s=\mathcal{D}(v(1))-\mathcal{D}(v(0))
$$

Therefore

$$
\operatorname{Dist}_{\mathcal{R}}\left(v_{0}, v_{1}\right)=\mathcal{D}\left(v_{1}\right)-\mathcal{D}\left(v_{0}\right), \quad \operatorname{Diss}_{B V}\left(v,\left[t_{a}, t_{b}\right]\right)=\mathcal{D}\left(v\left(t_{b}\right)\right)-\mathcal{D}\left(v\left(t_{a}\right)\right) .
$$

Now, let us see the role of the vanishing viscosity. The viscous dissipation takes the form $\mathcal{R}_{\varepsilon}(v, z)=\partial_{v} \mathcal{D}(v)+$ $(\varepsilon / 2)\|z\|^{2}$, therefore its Legendre transform is

$$
\mathcal{R}^{*}(v, \xi)=\frac{1}{2 \varepsilon}\left\|\xi-\partial_{v} \mathcal{D}(v)\right\|^{2} .
$$


As a consequence the contact potential reads

$$
\begin{aligned}
\mathfrak{p}(v, z, \xi) & =\inf _{\varepsilon>0}\left\{\mathcal{R}_{\varepsilon}(v, z)+\mathcal{R}_{\varepsilon}^{*}(v, \xi)\right\}=\partial_{v} \mathcal{D}(v)+\inf _{\varepsilon>0}\left\{(\varepsilon / 2)\|z\|^{2}+(1 / 2 \varepsilon)\left\|\xi-\partial_{v} \mathcal{D}(v)\right\|^{2}\right\} \\
& =\partial_{v} \mathcal{D}(v)+\|z\|\left\|\xi-\partial_{v} \mathcal{D}(v)\right\| .
\end{aligned}
$$

In particular $\mathfrak{p}\left(v, v^{\prime},-\partial_{v} \mathcal{E}(t, v)\right)=\partial_{v} \mathcal{D}(v)+\left\|v^{\prime}\right\|\left\|-\partial_{v} \mathcal{F}(t, v)\right\|$ and the Finsler distance becomes

$$
\begin{aligned}
\operatorname{Dist}_{\mathfrak{p}}\left(t, v_{0}, v_{1}\right) & =\inf \left\{\int_{0}^{1} \partial_{v} \mathcal{D}(v)+\left\|v^{\prime}\right\|\left\|-\partial_{v} \mathcal{F}(t, v)\right\| \mathrm{d} s: v \in W^{1,1}([0,1], \mathcal{V}), v(0)=v_{0}, v(1)=v_{1}\right\} \\
& =\mathcal{D}\left(v_{1}\right)-\mathcal{D}\left(v_{0}\right)+\inf \left\{\int_{0}^{1}\left\|v^{\prime}\right\|\left\|-\partial_{v} \mathcal{F}(t, v)\right\| \mathrm{d} s: v \in W^{1,1}([0,1], \mathcal{V}), v(0)=v_{0}, v(1)=v_{1}\right\} .
\end{aligned}
$$

Note that $-d_{s} \mathcal{F}(t, v(s))=-\partial_{v} \mathcal{F}(t, v(s))\left[v^{\prime}(s)\right] \leq\left\|v^{\prime}(s)\right\|\left\|\partial_{v} \mathcal{F}(t, v(s))\right\|$ and that equality holds if and only if $v^{\prime}(s) \in \operatorname{argmin}\left\{\partial_{v} \mathcal{F}(t, v(s))[\phi]:\|\phi\|=\left\|v^{\prime}(s)\right\|\right\}$. Hence, if there exists $v \in W^{1,1}([0,1], \mathcal{V})$ such that $v(0)=v_{0}$ and $v(1)=v_{1}$ and such that $v^{\prime}(s) \in \operatorname{argmin}\left\{\partial_{v} \mathcal{F}(t, v(s))[\phi]:\|\phi\|=\left\|v^{\prime}(s)\right\|\right\}$ a.e. in $[0,1]$ then we can write

$$
\inf \left\{\int_{0}^{1}\left\|v^{\prime}(s)\right\|\left\|-\partial_{v} \mathcal{F}(t, v(s))\right\| \mathrm{d} s\right\}=-\int_{0}^{1} d_{s} \mathcal{F}(t, v(s)) \mathrm{d} s=\mathcal{F}\left(t, v_{0}\right)-\mathcal{F}\left(t, v_{1}\right) .
$$

Thus, the term concentrated on the jumps becomes

$$
\begin{aligned}
\operatorname{Dist}_{\mathfrak{p}}\left(s, v(s), v\left(s^{+}\right)\right)-\operatorname{Dist}_{\mathcal{R}}\left(v(s), v\left(s^{+}\right)\right)= & \mathcal{D}\left(v\left(s^{+}\right)\right)-\mathcal{D}(v(s))+\mathcal{F}(t, v(s))-\mathcal{F}\left(t, v\left(s^{+}\right)\right) \\
& -\mathcal{D}\left(v\left(s^{+}\right)\right)+\mathcal{D}(v(s)) \\
= & \mathcal{F}(t, v(s))-\mathcal{F}\left(t, v\left(s^{+}\right)\right) .
\end{aligned}
$$

Thus $(\bar{E})$ reads: for every $t$ it holds

$$
\mathcal{E}(t, v(t))+\mathcal{D}(v(t))-\mathcal{D}\left(v_{0}\right)+\sum_{s \in J(v) \cap\left[t_{0}, t\right)} \mathcal{F}(s, v(s))-\mathcal{F}\left(s, v\left(s^{+}\right)\right)=\mathcal{E}\left(t_{0}, v_{0}\right)+\int_{t_{0}}^{t} \partial_{t} \mathcal{E}(s, v(s)) \mathrm{d} s .
$$

Rearranging the terms and writing $\partial_{t} \mathcal{E}(t, v)=\partial_{t} \mathcal{F}(t, v)$ we get

$$
\mathcal{F}(t, v(t))+\sum_{s \in J(v) \cap\left[t_{0}, t\right)} \mathcal{F}(s, v(s))-\mathcal{F}\left(s, v\left(s^{+}\right)\right)=\mathcal{F}\left(t_{0}, v_{0}\right)+\int_{t_{0}}^{t} \partial_{t} \mathcal{F}(s, v(s)) \mathrm{d} s .
$$

Therefore, in our setting we will say that a (left-continuous) trajectory $v \in B V([0,1], \mathcal{V})$ with $v\left(t_{0}\right)=v_{0}$ is a $B V$ solution if

$(\bar{S})$ for every $t \in\left(t_{0}, t_{1}\right]$ it holds

$$
\partial_{v} \mathcal{F}(t, v(t))=0,
$$

$(\bar{E})$ for every $t \in\left[t_{0}, t_{1}\right]$ it holds

$$
\mathcal{F}(t, v(t))+\sum_{s \in J(v) \cap\left[t_{0}, t\right)} \mathcal{F}(s, v(s))-\mathcal{F}\left(s, v\left(s^{+}\right)\right)=\mathcal{F}\left(t_{0}, v_{0}\right)+\int_{t_{0}}^{t} \partial_{t} \mathcal{F}(s, v(s)) \mathrm{d} s .
$$


Let us see how the conditions $\left(S^{\prime}\right)$ and $\left(E^{\prime}\right)$ of the parametrized solution correspond to $(\bar{S})$ and $(\bar{E})$. For convenience, we bring to mind that a Lipschitz map $(t, v):[0, \mathcal{T}) \rightarrow\left[t_{0}, t_{1}\right] \times \mathcal{V}$ with $(t(0), v(0))=\left(t_{0}, v_{0}\right)$, $0 \leq t^{\prime} \leq 1,\left\|v^{\prime}\right\| \leq 1$ is a parametrization of a quasi-static evolution if

$\left(S^{\prime}\right)$ for every $\tau$ with $t^{\prime}(\tau)>0$ it holds

$$
\left\|\partial_{v} \mathcal{F}(t(\tau), v(\tau))\right\|=0
$$

$\left(E^{\prime}\right)$ for every $\tau$ it holds

$$
\mathcal{F}(t(\tau), v(\tau))+\int_{0}^{\tau}\left\|\partial_{v} \mathcal{F}(t(s), v(s))\right\| \mathrm{d} s=\mathcal{F}\left(t_{0}, v_{0}\right)+\int_{0}^{\tau} \partial_{t} \mathcal{F}(t(s), v(s)) t^{\prime}(s) \mathrm{d} s .
$$

It is not restrictive to consider the map $\tau \mapsto t(\tau)$ to be surjective but it is not possible to assume that it is invertible; however, using monotonicity, we can define for $s \in\left[t_{0}, t_{1}\right]$

$$
\tau_{-1}(s)=\inf \{\tau: t(\tau)=s\} .
$$

It is not difficult to check that $\tau_{-1}$ is strictly increasing, bounded, left-continuous and of course that $t \circ \tau_{-1}(s)=s$. Being $\tau \mapsto v(\tau)$ Lipschitz continuous, it follows that the function $w(t)=v \circ \tau_{-1}(t)$ is left-continuous and of class $B V\left(\left[t_{0}, t_{1}\right], \mathcal{V}\right)$. Let us check that $w$ is indeed a $B V$ solution.

Consider the set $I_{+}=\left\{s \in\left[t_{0}, t_{1}\right]: s=t(\tau)\right.$ with $\left.t^{\prime}(\tau)>0\right\}$. Note that the set $I_{+}$is dense in $\left[t_{0}, t_{1}\right](e . g$. by a contradiction argument). If $\bar{t} \in I_{+}$and $\bar{t}=t(\bar{\tau})$ then, being $t^{\prime}(\bar{\tau})>0$, we have $\tau_{-1}(\bar{t})=\bar{\tau}$. By $\left(S^{\prime}\right)$ for every $\bar{t} \in I_{+}$it holds $\partial_{v} \mathcal{F}(\bar{t}, w(\bar{t}))=\partial_{v} \mathcal{F}(t(\bar{\tau}), v(\bar{\tau}))=0$. The density of $I_{+}$together with the continuity of $\partial_{v} \mathcal{F}$ and the left-continuity of $w$ lead to $(\bar{S})$.

It remains to show that $(\bar{E})$ follows from $\left(E^{\prime}\right)$. Fix $\bar{t} \in I_{+}$and let $\bar{t}=t(\bar{\tau})$. We claim that

$$
\int_{0}^{\bar{\tau}}\left\|\partial_{v} \mathcal{F}(t(\tau), v(\tau))\right\| \mathrm{d} \tau=\sum_{s \in J\left(\tau_{-1}\right) \cap\left[t_{0}, \bar{t}\right)} \int_{\tau_{-1}\left(s^{-}\right)}^{\tau_{-1}\left(s^{+}\right)}\left\|\partial_{v} \mathcal{F}(t(\tau), v(\tau))\right\| \mathrm{d} \tau .
$$

Let $\tau \in[0, \bar{\tau}]$. If there exists a sequence $\tau_{n} \rightarrow \tau$ with $t^{\prime}\left(\tau_{n}\right)>0$ then $\left\|\partial_{v} \mathcal{F}\left(t\left(\tau_{n}\right), v\left(\tau_{n}\right)\right)\right\|=0$ and by continuity $\left\|\partial_{v} \mathcal{F}(t(\tau), v(\tau))\right\|=0$. Thus, to evaluate the integral on the left hand side, it is sufficient to consider those values $\tau$ for which $t^{\prime}=0$ in a neighbourhood of $\tau$. In this case $s=t(\tau)$ is a discontinuity point for $\tau_{-1}$ and $\tau \in\left(\tau_{-1}\left(s^{-}\right), \tau_{-1}\left(s^{+}\right)\right)$. Thus (3.1) is proved. Moreover,

$$
\begin{aligned}
\int_{\tau_{-1}\left(s^{-}\right)}^{\tau_{-1}\left(s^{+}\right)}\left\|\partial_{v} \mathcal{F}(t(\tau), v(\tau))\right\| \mathrm{d} \tau & =\int_{\tau_{-1}\left(s^{-}\right)}^{\tau_{-1}\left(s^{+}\right)}-\partial_{v} \mathcal{F}(s, v(\tau))\left[v^{\prime}(\tau)\right] \mathrm{d} \tau \\
& =\mathcal{F}\left(s, v \circ \tau_{-1}\left(s^{-}\right)\right)-\mathcal{F}\left(s, v \circ \tau_{-1}\left(s^{+}\right)\right)=\mathcal{F}(s, w(s))-\mathcal{F}\left(s, w\left(s^{+}\right)\right) .
\end{aligned}
$$

Hence

$$
\int_{0}^{\bar{\tau}}\left\|\partial_{v} \mathcal{F}(t(\tau), v(\tau))\right\| \mathrm{d} \tau=\sum_{s \in J\left(\tau_{-1}\right) \cap\left[t_{0}, \bar{t}\right)} \mathcal{F}(s, w(s))-\mathcal{F}\left(s, w\left(s^{+}\right)\right) .
$$

Note that if $s \in J(w)$ then (being $v$ continuous) $s \in J\left(\tau_{-1}\right)$. Moreover, if $s \in J\left(\tau_{-1}\right) \backslash J(w)$ then $\mathcal{F}(s, w(s))=$ $\mathcal{F}\left(s, w\left(s^{-}\right)\right)=\mathcal{F}\left(s, w\left(s^{+}\right)\right)$. In conclusion we can write that

$$
\int_{0}^{\bar{\tau}}\left\|\partial_{v} \mathcal{F}(t(\tau), v(\tau))\right\| \mathrm{d} \tau=\sum_{s \in J(w) \cap\left[t_{0}, \bar{t}\right)} \mathcal{F}(s, w(s))-\mathcal{F}\left(s, w\left(s^{+}\right)\right) .
$$

Next, by a change of variable we obtain the integral of the power

$$
\int_{t_{0}}^{\bar{t}} \partial_{t} \mathcal{F}(t, w(t)) \mathrm{d} t=\int_{0}^{\bar{\tau}} \partial_{t} \mathcal{F}(t(\tau), v(\tau)) t^{\prime}(\tau) \mathrm{d} \tau .
$$

Since $\bar{t} \in I_{+}$we have $\mathcal{F}(t(\bar{\tau}), v(\bar{\tau}))=\mathcal{F}(\bar{t}, w(\bar{t}))$ and thus, using (3.2) and (3.3), we get that $(\bar{E})$ holds for every $t \in I_{+}$. If $\bar{t} \notin I_{+}$it is sufficient to use again the continuity of $\mathcal{F}, \partial_{t} \mathcal{F}, \partial_{v} \mathcal{F}$ together with the left-continuity of $w$ and the density of $I_{+}$in $\left[t_{0}, t_{1}\right]$. 


\section{Existence By A PARAMETRIZED MINIMIZING MOVEMENT}

\subsection{Incremental problem}

In this section we prove the existence result following the discretization used in [16]. We will assume that $\mathcal{F}$ satisfies the hypotheses of Theorem 2.2. Let $\Delta \tau_{n} \rightarrow 0^{+}$. For every $n \in \mathbb{N}$ we define a discrete in time evolution by means of constrained incremental minimization problems. Set the initial conditions $t_{n, 0}=t_{0}$ and $v_{n, 0}=v_{0}$; known $t_{n, k}<t_{1}$ and $v_{n, k}$ define $t_{n, k+1}$ and $v_{n, k+1}$ as

$$
\left\{\begin{array}{l}
v_{n, k+1} \in \operatorname{argmin}\left\{\mathcal{F}\left(t_{n, k}, v\right):\left\|v-v_{n, k}\right\| \leq \Delta \tau_{n}\right\}, \\
t_{n, k+1}=t_{n, k}+\left(\Delta \tau_{n}-\left\|v_{n, k+1}-v_{n, k}\right\|\right) .
\end{array}\right.
$$

Existence of a minimizer follows by the direct method of the calculus of variations: weak compactness of the closed ball $\left\|v-v_{n, k}\right\| \leq \Delta \tau_{n}$ is a consequence of $\mathcal{V}$ being reflexive while the weak lower semi-continuity of $\mathcal{F}\left(t_{n, k}, \cdot\right)$ is assumed in (2.3). (Note that a similar incremental problem, actually without parametrization, appears in [15], Example 1.3). Let $\bar{k}_{n}=\sup \left\{k: t_{n, k}<t_{1}\right\}$ where $\bar{k}_{n} \in \mathbb{N} \cup\{\infty\}$ (the incremental construction can be finite or infinite). Note that $0 \leq t_{n, k+1}-t_{n, k} \leq \Delta \tau_{n}$ and thus $\bar{k}_{n} \geq\left(t_{1}-t_{0}\right) / \Delta \tau_{n}$.

Next, let $\tau_{n, k}=k \Delta \tau_{n}$ for $0 \leq k \leq \bar{k}_{n}$ and denote $\mathcal{T}_{n}=\bar{k}_{n} \Delta \tau_{n} \geq\left(t_{1}-t_{0}\right)$. Now we consider the affine interpolation of $t_{n, k}$ and $v_{n, k}$ in the points $\tau_{n, k}$; in this way we define the discrete evolutions $\left(t_{n}, v_{n}\right):\left[0, \mathcal{T}_{n}\right) \rightarrow$ $\left[t_{0}, t_{1}\right] \times \mathcal{V}$. It is important to remark that the map $\left(t_{n}, v_{n}\right)$ is Lipschitz continuous with $t_{n}^{\prime} \geq 0$ and $t_{n}^{\prime}+\left\|v_{n}^{\prime}\right\|=1$ a.e. in $\left[0, \mathcal{T}_{n}\right)$.

Now, let us see the two properties which will provide the base to get $\left(S^{\prime}\right)$ and $\left(E^{\prime}\right)$ in the limit as $\Delta \tau_{n} \rightarrow 0^{+}$.

Proposition 4.1. If $t_{n, k+1}>t_{n, k}$ then $v_{n, k+1}$ satisfies the equilibrium condition

$$
\left\|\partial_{v} \mathcal{F}\left(t_{n, k}, v_{n, k+1}\right)\right\|=0 .
$$

Proof. If $t_{n, k+1}>t_{n, k}$ then by (4.1) we have $\left\|v_{n, k+1}-v_{n, k}\right\|<\Delta \tau_{n}$. Since $v_{n, k+1}$ is a minimizer in the open ball $\left\|v-v_{n, k}\right\|<\Delta \tau_{n}$ the Euler-Lagrange equation (4.2) holds.

Proposition 4.2. The following incremental energy estimate holds

$$
\begin{aligned}
\mathcal{F}\left(t_{n, k+1}, v_{n, k+1}\right) \leq & \mathcal{F}\left(t_{n, k}, v_{n, k}\right)-\int_{\tau_{n, k}}^{\tau_{n, k+1}}\left\|\partial_{v} \mathcal{F}\left(t_{n, k}, v_{n}(\tau)\right)\right\| \mathrm{d} \tau \\
& +\int_{\tau_{n, k}}^{\tau_{n, k+1}} \partial_{t} \mathcal{F}\left(t_{n}(\tau), v_{n}(\tau)\right) t_{n}^{\prime}(\tau) \mathrm{d} \tau+3 \mathcal{C}\left(\Delta \tau_{n}\right) \Delta \tau_{n},
\end{aligned}
$$

where $\mathcal{C}$ is the modulus of continuity appearing in (2.6).

Proof. Given $\left(t_{n, k}, v_{n, k}\right)$ let

$$
\phi_{n, k} \in \operatorname{argmin}\left\{\partial_{v} \mathcal{F}\left(t_{n, k}, v_{n, k}\right)[\phi]:\|\phi\| \leq 1\right\} .
$$

Now, we write

$$
\begin{aligned}
\mathcal{F}\left(t_{n, k+1}, v_{n, k+1}\right)= & \mathcal{F}\left(t_{n, k}, v_{n, k+1}\right)+\int_{t_{n, k}}^{t_{n, k+1}} \partial_{t} \mathcal{F}\left(t, v_{n, k+1}\right) \mathrm{d} t \\
\leq & \mathcal{F}\left(t_{n, k}, v_{n, k}+\left(\tau_{n, k+1}-\tau_{n, k}\right) \phi_{n, k}\right)+\int_{\tau_{n, k}}^{\tau_{n, k+1}} \partial_{t} \mathcal{F}\left(t_{n}(\tau), v_{n, k+1}\right) t_{n}^{\prime}(\tau) \mathrm{d} \tau \\
= & \mathcal{F}\left(t_{n, k}, v_{n, k}\right)+\int_{\tau_{n, k}}^{\tau_{n, k+1}} \partial_{v} \mathcal{F}\left(t_{n, k}, v_{n, k}+\left(\tau-\tau_{n, k}\right) \phi_{n, k}\right)\left[\phi_{n, k}\right] \mathrm{d} \tau \\
& +\int_{\tau_{n, k}}^{\tau_{n, k+1}} \partial_{t} \mathcal{F}\left(t_{n}(\tau), v_{n, k+1}\right) t_{n}^{\prime}(\tau) \mathrm{d} \tau
\end{aligned}
$$


We consider separately the two integrals on the right hand side. For every $\tau \in\left[\tau_{n, k}, \tau_{n, k+1}\right]$ by (2.6) we can write

$$
\begin{aligned}
\partial_{v} \mathcal{F}\left(t_{n, k}, v_{n, k}+\left(\tau-\tau_{n, k}\right) \phi_{n, k}\right)\left[\phi_{n, k}\right] & \leq \partial_{v} \mathcal{F}\left(t_{n, k}, v_{n, k}\right)\left[\phi_{n, k}\right]+\mathcal{C}\left(\Delta \tau_{n}\right) \\
& \leq-\left\|\partial_{v} \mathcal{F}\left(t_{n, k}, v_{n, k}\right)\right\|+\mathcal{C}\left(\Delta \tau_{n}\right) \\
& \leq-\left\|\partial_{v} \mathcal{F}\left(t_{n, k}, v_{n}(\tau)\right)\right\|+2 \mathcal{C}\left(\Delta \tau_{n}\right)
\end{aligned}
$$

Thus

$$
\int_{\tau_{n, k}}^{\tau_{n, k+1}} \partial_{v} \mathcal{F}\left(t_{n, k}, v_{n, k}+\left(\tau-\tau_{n, k}\right) \phi_{n, k}\right)\left[\phi_{n, k}\right] \mathrm{d} \tau \leq-\int_{\tau_{n, k}}^{\tau_{n, k+1}}\left\|\partial_{v} \mathcal{F}\left(t_{n, k}, v_{n}(\tau)\right)\right\| \mathrm{d} \tau+2 \mathcal{C}\left(\Delta \tau_{n}\right) \Delta \tau_{n}
$$

Similarly, again by (2.6), for every $\tau \in\left[\tau_{n, k}, \tau_{n, k+1}\right]$

$$
\partial_{t} \mathcal{F}\left(t_{n}(\tau), v_{n, k+1}\right) \leq \partial_{t} \mathcal{F}\left(t_{n}(\tau), v_{n}(\tau)\right)+\mathcal{C}\left(\Delta \tau_{n}\right) .
$$

As $0 \leq t_{n}^{\prime} \leq 1$ we get

$$
\int_{\tau_{n, k}}^{\tau_{n, k+1}} \partial_{t} \mathcal{F}\left(t_{n}(\tau), v_{n, k+1}\right) t_{n}^{\prime}(\tau) \mathrm{d} \tau \leq \int_{\tau_{n, k}}^{\tau_{n, k+1}} \partial_{t} \mathcal{F}\left(t_{n}(\tau), v_{n}(\tau)\right) t_{n}^{\prime}(\tau) \mathrm{d} \tau+\mathcal{C}\left(\Delta \tau_{n}\right) \Delta \tau_{n},
$$

which concludes the proof.

\subsection{Compactness and convergence}

Proposition 4.3. Let $\left(t_{n}, v_{n}\right):\left[0, \mathcal{T}_{n}\right) \rightarrow\left[t_{0}, t_{1}\right] \times \mathcal{V}$ be given by Section 4.1. Let $0<\mathcal{T}<\liminf { }_{n} \mathcal{T}_{n}$. There exists a subsequence (not relabelled) such that $t_{n} \stackrel{*}{\rightarrow} t$ in $W^{1, \infty}(0, \mathcal{T})$ and $v_{n} \stackrel{*}{\rightarrow} v$ in $W^{1, \infty}(0, \mathcal{T} ; \mathcal{V})$. In particular $t_{n}\left(\tau_{n}\right) \rightarrow t(\tau)$ and $v_{n}\left(\tau_{n}\right) \rightarrow v(\tau)$ in $\mathcal{V}$ if $\tau_{n} \rightarrow \tau$. Moreover $0 \leq t^{\prime} \leq 1$ and $\left\|v^{\prime}\right\| \leq 1$ a.e. in $(0, \mathcal{T})$

Proof. As $\mathcal{T}_{n} \geq\left(t_{1}-t_{0}\right)$ we have $\liminf \operatorname{in}_{n} \mathcal{T}_{n}>0$. Then, for $n$ sufficiently large $\mathcal{T}_{n}>\mathcal{T}$ and we can consider $\left(t_{n}, v_{n}\right)$ to be defined in $(0, \mathcal{T})$. Since $\left|t_{n}^{\prime}(\tau)\right| \leq 1$ there exists a subsequence (not relabelled) with $t_{n} \stackrel{*}{\rightarrow} t$ in $W^{1, \infty}(0, \mathcal{T})$. As $t_{n}$ is non-decreasing the limit $t$ is non-decreasing, moreover we have $0 \leq t^{\prime} \leq 1$.

Being $\mathcal{V}$ reflexive and separable its dual $\mathcal{V}^{\prime}$ is reflexive and separable, thus the space $L^{1}\left(0, \mathcal{T} ; \mathcal{V}^{\prime}\right)$ is separable and its dual is $L^{\infty}(0, \mathcal{T} ; \mathcal{V})$. The sequence $v_{n}^{\prime}$ is bounded in $L^{\infty}(0, \mathcal{T} ; \mathcal{V})$ and thus there exists a subsequence (not relabelled) such that $v_{n}^{\prime} \stackrel{*}{\rightarrow} v^{\prime}$ in $L^{\infty}(0, \mathcal{T} ; \mathcal{V})$. Let $\tau_{n} \rightarrow \tau$. Denoting by $\langle$,$\rangle the duality pairing between \mathcal{V}$ and $\mathcal{V}^{\prime}$ and by $($,$) the corresponding duality between L^{1}\left(0, \mathcal{T} ; \mathcal{V}^{\prime}\right)$ and $L^{\infty}(0, \mathcal{T} ; \mathcal{V})$, we can write

$$
\left\langle v_{n}\left(\tau_{n}\right), \psi\right\rangle=\left\langle v_{0}+\int_{0}^{\tau_{n}} v_{n}^{\prime}(s) \mathrm{d} s, \psi\right\rangle=\left\langle v_{0}, \psi\right\rangle+\int_{0}^{\tau_{n}}\left\langle v_{n}^{\prime}(s), \psi\right\rangle \mathrm{d} s=\left\langle v_{0}, \psi\right\rangle+\left(v_{n}^{\prime}, \psi \chi_{\left(0, \tau_{n}\right)}\right)
$$

from which it follows that $\left\langle v_{n}\left(\tau_{n}\right), \psi\right\rangle \rightarrow\langle v(\tau), \psi\rangle$ for every $\psi \in \mathcal{V}^{\prime}$. By the lower semi-continuity of the norm with respect to weak* convergence in $L^{\infty}(0, \mathcal{T} ; \mathcal{V})$ it follows that $\left\|v^{\prime}(\tau)\right\| \leq 1$ for a.e. $\tau \in(0, \mathcal{T})$.

Theorem 4.4. Let $\left(t_{n}, v_{n}\right)$ and $(t, v)$ be as in Proposition 4.3; then $(t, v)$ is a quasi-static evolution in the sense of Definition 2.1, i.e. it satisfies $(t(0), v(0))=\left(t_{0}, v_{0}\right), 0 \leq t^{\prime} \leq 1,\left\|v^{\prime}\right\| \leq 1$, and

$\left(S^{\prime}\right)$ for every $\tau>0$ with $t^{\prime}(\tau)>0$ it holds

$$
\left\|\partial_{v} \mathcal{F}(t(\tau), v(\tau))\right\|=0
$$

$\left(E^{\prime}\right)$ for every $\tau$ it holds

$$
\mathcal{F}(t(\tau), v(\tau))=\mathcal{F}\left(t_{0}, v_{0}\right)-\int_{0}^{\tau}\left\|\partial_{v} \mathcal{F}(t(s), v(s))\right\| \mathrm{d} s+\int_{0}^{\tau} \partial_{t} \mathcal{F}(t(s), v(s)) t^{\prime}(s) \mathrm{d} s .
$$


Proof. If $t^{\prime}(\tau)>0$ then $t(\tau)<t(\tau+\delta)$ for every $\delta>0$, further, since $t_{n}$ converges to $t$ pointwise, for every $n$ sufficiently large there exists an index $k$ (depending on $n$ ) such that $\tau<\tau_{n, k}<\tau+\delta$ and $t_{n, k}=t_{n}\left(\tau_{n, k}\right)<$ $t_{n}\left(\tau_{n, k+1}\right)=t_{n, k+1}$. Since $t_{n, k}<t_{n, k+1}$ by Lemma 4.1 we get

$$
\left\|\partial_{v} \mathcal{F}\left(t_{n, k}, v_{n, k+1}\right)\right\|=0 .
$$

By the arbitrarity of $\delta$ it follows that there exists a sequence $k_{n}$ with $\tau_{n, k_{n}} \rightarrow \tau$ such that $t_{n, k_{n}}<t_{n, k_{n}+1}$ and $\left\|\partial_{v} \mathcal{F}\left(t_{n, k_{n}}, v_{n, k_{n}+1}\right)\right\|=0$. By Proposition 4.3 we known that $t_{n, k_{n}}=t_{n}\left(\tau_{n, k_{n}}\right) \rightarrow t(\tau)$ and that $v_{n, k_{n}+1}=$ $v_{n}\left(\tau_{n, k_{n}+1}\right) \rightarrow v(\tau)$ weakly in $\mathcal{V}$. Then, by $(2.4)$

$$
\left\|\partial_{v} \mathcal{F}(t(\tau), v(\tau))\right\| \leq \liminf _{n}\left\|\partial_{v} \mathcal{F}\left(t_{n, k_{n}}, v_{n, k_{n}+1}\right)\right\|=0
$$

which is $\left(S^{\prime}\right)$.

For the proof of $\left(E^{\prime}\right)$ it is more convenient to have $\tau$ as the integration variable, so we will show that for every $s$

$$
\mathcal{F}(t(s), v(s))=\mathcal{F}\left(t_{0}, v_{0}\right)-\int_{0}^{s}\left\|\partial_{v} \mathcal{F}(t(\tau), v(\tau))\right\| \mathrm{d} \tau+\int_{0}^{s} \partial_{t} \mathcal{F}(t(\tau), v(\tau)) t^{\prime}(\tau) \mathrm{d} \tau .
$$

For every $n \in \mathbb{N}$ let $k \in \mathbb{N}$ (depending on $n$ ) such that $s \in\left[\tau_{n, k}, \tau_{n, k+1}\right.$ ). Iterating the incremental energy estimate of Proposition 4.2 yields

$$
\begin{aligned}
\mathcal{F}\left(t_{n, k}, v_{n, k}\right) \leq & \mathcal{F}\left(t_{0}, v_{0}\right)-\sum_{m=0}^{k-1} \int_{\tau_{n, m}}^{\tau_{n, m+1}}\left\|\partial_{v} \mathcal{F}\left(t_{n, m}, v_{n}(\tau)\right)\right\| \mathrm{d} \tau \\
& +\int_{0}^{\tau_{n, k}} \partial_{t} \mathcal{F}\left(t_{n}(\tau), v_{n}(\tau)\right) t_{n}^{\prime}(\tau) \mathrm{d} \tau+3 \mathcal{C}\left(\Delta \tau_{n}\right) \mathcal{T}
\end{aligned}
$$

Taking the limsup we get

$$
\begin{aligned}
\limsup _{n} \mathcal{F}\left(t_{n, k}, v_{n, k}\right) \leq & \mathcal{F}\left(t_{0}, v_{0}\right)-\liminf _{n} \sum_{m=0}^{k-1} \int_{\tau_{n, m}}^{\tau_{n, m+1}}\left\|\partial \mathcal{F}\left(t_{n, m}, v_{n}(\tau)\right)\right\| \mathrm{d} \tau \\
& +\underset{n}{\limsup } \int_{0}^{\tau_{n, k}} \partial_{t} \mathcal{F}\left(t_{n}(\tau), v_{n}(\tau)\right) t_{n}^{\prime}(\tau) \mathrm{d} \tau
\end{aligned}
$$

Given $\tau$, let $m$ (depending on $n$ and $\tau$ ) with $\tau_{n, m} \leq \tau<\tau_{n, m+1}$; since $t_{n, m}=t\left(\tau_{n, m}\right) \rightarrow t(\tau)$ and $v_{n}(\tau) \rightarrow v(\tau)$ by (2.4) we get

$$
\left\|\partial_{v} \mathcal{F}(t(\tau), v(\tau))\right\| \leq \liminf _{n}\left\|\partial_{v} \mathcal{F}\left(t_{n, m}, v_{n}(\tau)\right)\right\|
$$

and by Fatou's Lemma we get

$$
\int_{0}^{s}\left\|\partial_{v} \mathcal{F}(t(\tau), v(\tau))\right\| \mathrm{d} \tau \leq \liminf _{n} \sum_{m=0}^{k-1} \int_{\tau_{n, m}}^{\tau_{n, m+1}}\left\|\partial_{v} \mathcal{F}\left(t_{n, m}, v_{n}(\tau)\right)\right\| \mathrm{d} \tau .
$$

By (2.5) we know that $\partial_{t} \mathcal{F}\left(t_{n}(\tau), v_{n}(\tau)\right)$ converges to $\partial_{t} \mathcal{F}(t(\tau), v(\tau))$. Since $\left\|v_{n}^{\prime}\right\| \leq 1$ it follows that $\| v_{n}(\tau)-$ $v_{0} \| \leq \mathcal{T}$ for every $\tau \in(0, \mathcal{T})$. Then $(2.6)$ implies that $\partial_{t} \mathcal{F}\left(t_{n}(\cdot), v_{n}(\cdot)\right)$ is uniformly bounded from above; therefore by dominated convergence $\partial_{t} \mathcal{F}\left(t_{n}(\cdot), v_{n}(\cdot)\right)$ converge to $\partial_{t} \mathcal{F}(t(\cdot), v(\cdot))$ strongly in $L^{1}(0, \mathcal{T})$. We already know from Proposition 4.3 that $t_{n}^{\prime} \stackrel{*}{\rightarrow} t^{\prime}$ in $L^{\infty}(0, \mathcal{T})$. As a consequence

$$
\lim _{n} \int_{0}^{\tau_{n, k}} \partial_{t} \mathcal{F}\left(t_{n}(\tau), v_{n}(\tau)\right) t_{n}^{\prime}(\tau) \mathrm{d} \tau=\int_{0}^{s} \partial_{t} \mathcal{F}(t(\tau), v(\tau)) t^{\prime}(\tau) \mathrm{d} \tau
$$


Therefore

$$
\limsup _{n} \mathcal{F}\left(t_{n, k}, v_{n, k}\right) \leq \mathcal{F}\left(t_{0}, v_{0}\right)-\int_{0}^{s}\left\|\partial_{v} \mathcal{F}(t(\tau), v(\tau))\right\| \mathrm{d} \tau+\int_{0}^{s} \partial_{t} \mathcal{F}(t(\tau), v(\tau)) t^{\prime}(\tau) \mathrm{d} \tau
$$

Since $\left\|v^{\prime}\right\| \leq 1$ we can write

$$
-\left\|\partial_{v} \mathcal{F}(t(\tau), v(\tau))\right\| \leq \partial_{v} \mathcal{F}(t(\tau), v(\tau))\left[v^{\prime}(\tau)\right]
$$

and by the chain rule

$$
\begin{aligned}
\limsup _{n} \mathcal{F}\left(t_{n, k}, v_{n, k}\right) & \leq \mathcal{F}\left(t_{0}, v_{0}\right)-\int_{0}^{s}\left\|\partial_{v} \mathcal{F}(t(\tau), v(\tau))\right\| \mathrm{d} \tau+\int_{0}^{s} \partial_{t} \mathcal{F}(t(\tau), v(\tau)) t^{\prime}(\tau) \mathrm{d} \tau \\
& \leq \mathcal{F}\left(t_{0}, v_{0}\right)+\int_{0}^{s} \partial_{v} \mathcal{F}(t(\tau), v(\tau))\left[v^{\prime}(\tau)\right] \mathrm{d} \tau+\int_{0}^{s} \partial_{t} \mathcal{F}(t(\tau), v(\tau)) t^{\prime}(\tau) \mathrm{d} \tau \\
& \leq \mathcal{F}\left(t_{0}, v_{0}\right)+\int_{0}^{s} d_{\tau} \mathcal{F}(t(\tau), v(\tau)) \mathrm{d} \tau=\mathcal{F}(t(s), v(s)) .
\end{aligned}
$$

Thus $\limsup _{n} \mathcal{F}\left(t_{n, k}, v_{n, k}\right) \leq \mathcal{F}(t(s), v(s))$. On the other hand, $t_{n, k} \rightarrow t(s)$ and $v_{n, k} \rightarrow v(s)$ by Proposition 4.3, thus by (2.3) we can write $\mathcal{F}(t(s), v(s)) \leq \liminf _{n} \mathcal{F}\left(t_{n, k}, v_{n, k}\right)$. It follows that

$$
\limsup _{n} \mathcal{F}\left(t_{n, k}, v_{n, k}\right)=\lim _{n} \mathcal{F}\left(t_{n, k}, v_{n, k}\right)=\mathcal{F}(t(s), v(s))
$$

and all the inequalities in (4.4) become equalities, which gives $\left(E^{\prime}\right)$.

As a by-product of the previous results we get the convergence of energies, stated in the next Corollary.

Corollary 4.5. $\mathcal{F}\left(t_{n}(\tau), v_{n}(\tau)\right) \rightarrow \mathcal{F}(t(\tau), v(\tau))$ for every $\tau \in[0, \mathcal{T})$.

Proof. For $\tau \in\left[\tau_{n, k}, \tau_{n, k+1}\right)$ both $\left\|v_{n}(\tau)-v_{n, k}\right\| \leq \Delta \tau_{n}$ and $\left|t_{n}(\tau)-t_{n, k}\right| \leq \Delta \tau_{n}$ therefore by (4.5) together with the uniform continuity of $\mathcal{F}$ we get the pointwise convergence of the energy.

Finally, note that, without further assumptions, it is not obvious that the limit $(t, v)$ (provided by Prop. 4.3) is not the trivial evolution $(t(\tau), v(\tau))=\left(t_{0}, v_{0}\right)$. For instance, consider the energy

$$
\mathcal{F}\left(t_{0}, v\right)= \begin{cases}0 & \text { if }\|v\| \leq 1, \\ (\|v\|-1)^{2} & \text { otherwise }\end{cases}
$$

The unit ball is the set of minimizers of this functional. If $\Delta \tau_{n} \leq 1$ and if $v_{0}=0$ it is possible to choose a sequence $v_{n, k}$ with $\left\|v_{n, k}\right\| \leq \Delta \tau_{n}$ and with $\left\|v_{n, k+1}-v_{n, k}\right\|=\Delta \tau_{n}$. As a consequence $t_{n, k}=t_{0}$ and $v_{n, k} \rightarrow v_{0}$, therefore the limit is the trivial solution. In the applications irreversibility conditions are usually helpful to rule out this solution, see [16].

\section{Existence By A FORWARD EULER SCHEME}

\subsection{Incremental problem}

In applications and in numerical simulations sometimes it is not feasible to minimize (even locally) the energy of a non-convex function, as in the previous scheme, while it is more feasible to employ a forward scheme, based on descent directions. Let us see how to define the evolution in this way.

We will assume that $\mathcal{F}$ satisfies the hypotheses of Theorem 2.2. Let $\Delta \tau_{n}$ be positive with $\Delta \tau_{n} \rightarrow 0$ and denote again by $\tau$ the parametrization variable. Let $\tau_{n, k}=k \Delta \tau_{n}$. As before, we will provide a sequence converging to (the parametrization of) a quasi-static evolution, according to Definition 2.1. Let the initial conditions (for $\left.\tau_{n, 0}=0\right)$ be $t_{n, 0}=t_{0}$ and $v_{n, 0}=v_{0}$. Given $t_{n, k}$ and $v_{n, k}$ we will employ a further sequence, denoted by $v_{n, k, i}$ 
for $i \in \mathbb{N}$, in order to define $v_{n, k+1}$ and then $t_{n, k+1}$. For convenience we will also introduce a sequence $\tau_{n, k, i}$ which actually depends on $v_{n, k, i}$. Let $v_{n, k, 0}=v_{n, k}$ and $\tau_{n, k, 0}=\tau_{n, k}$; given $v_{n, k, i}$ and $\tau_{n, k, i}$ consider the steepest descent direction

$$
\phi_{n, k, i} \in \operatorname{argmin}\left\{\partial_{v} \mathcal{F}\left(t_{n, k}, v_{n, k, i}\right)[\phi]:\|\phi\| \leq 1\right\} .
$$

Note for future reference that the minimum value in (5.1) is non-positive. Existence of $\phi_{n, k, i}$ is straightforward. Uniqueness holds for instance when $\partial_{v} \mathcal{F}\left(t_{n, k}, v_{n, k, i}\right) \neq 0$ and $\mathcal{V}$ is uniformly convex: indeed, if $\partial_{v} \mathcal{F}\left(t_{n, k}, v_{n, k, i}\right) \neq$ 0 then $\left\|\phi_{n, k, i}\right\|=1$, thus if

$$
\zeta, \xi \in \operatorname{argmin}\left\{\partial_{v} \mathcal{F}\left(t_{n, k}, v_{n, k, i}\right)[\phi]:\|\phi\| \leq 1\right\}
$$

then by linearity $(\zeta+\xi) / 2$ is a minimizer and thus $\|(\zeta+\xi) / 2\|=1$; the uniform convexity of $\mathcal{V}$ implies that $\zeta=\xi$. On the contrary, if $\partial_{v} \mathcal{F}\left(t_{n, k}, v_{n, k, i}\right)[\phi]=0$ then every $\phi$ is a minimizer, in this case it is convenient to choose again a direction $\phi_{n, k, i}$ with $\left\|\phi_{n, k, i}\right\|=1$ in order to avoid any trouble in the following construction. In any case

$$
\partial_{v} \mathcal{F}\left(t_{n, k}, v_{n, k, i}\right)\left[\phi_{n, k, i}\right]=-\| \partial_{v} \mathcal{F}\left(t_{n, k}, v_{n, k, i}\right) .
$$

Once the direction $\phi_{n, k, i}$ is found the step $s_{n, k, i}$ is given by a gradient descent in the direction $\phi_{n, k, i}$. To this end, for $s \in\left[0, \Delta \tau_{n}-\left(\tau_{n, k, i}-\tau_{n, k}\right)\right]=\left[0, \Delta \tau_{n}-\left(\tau_{n, k, i}-\tau_{n, k, 0}\right)\right]$, let us introduce the function

$$
f(s)=\mathcal{F}\left(t_{n, k}, v_{n, k, i}+s \phi_{n, k, i}\right)
$$

and the associated ODE

$$
\left\{\begin{array}{l}
s^{\prime}(r)=\left[-f^{\prime}(s(r))\right]_{+}, \\
s(0)=0,
\end{array}\right.
$$

where $[\cdot]_{+}$denotes the positive part (the independent variable $r$ in the ODE is purely auxiliary and has no physical meaning). Here, in analogy with curves of maximal slope, the positive part in (5.2) guarantees that the solution $s$ is non-negative and non-decreasing and that the energy $f(s(r))$ is thus non-increasing. Moreover, since

$$
f^{\prime}(s)=\partial_{v} \mathcal{F}\left(t_{n, k}, v_{n, k, i}+s \phi_{n, k, i}\right)\left[\phi_{n, k, i}\right]
$$

it follows, by the assumptions on $\partial_{v} \mathcal{F}$, that the right hand side in (5.2) is continuous and bounded, therefore there exists a solution $s$ of the ODE. Being $s$ non-decreasing it makes sense to take $s_{n, k, i}=\sup _{r} s(r) \leq \Delta \tau_{n}-$ $\left(\tau_{n, k, i}-\tau_{n, k}\right)$. In particular, it may happen that either the solution $s$ reaches the upper bound $\Delta \tau_{n}-\left(\tau_{n, k, i}-\tau_{n, k}\right)$ in a finite interval or that $s$ exists in $[0,+\infty)$. In the latter case

$$
\lim _{r \rightarrow+\infty} s^{\prime}(r)=\lim _{r \rightarrow+\infty}\left[-f^{\prime}(s(r))\right]_{+}=\left[-f^{\prime}\left(s_{n, k, i}\right)\right]_{+}=0 .
$$

On the other hand $f(s(r))$ is non-increasing, hence $f^{\prime}(s(r)) \leq 0$ and

$$
\lim _{r \rightarrow+\infty} f^{\prime}(s(r))=f^{\prime}\left(s_{n, k, i}\right) \leq 0 .
$$

Therefore, if $s_{n, k, i}<\Delta \tau_{n}-\left(\tau_{n, k, i}-\tau_{n, k}\right)$ then the solution $s$ is defined in $[0,+\infty)$ and

$$
f^{\prime}\left(s_{n, k, i}\right)=\partial_{v} \mathcal{F}\left(t_{n, k}, v_{n, k, i}+s_{n, k, i} \phi_{n, k, i}\right)\left[\phi_{n, k, i}\right]=0 .
$$

At this point we can define

$$
\begin{aligned}
& v_{n, k, i+1}=v_{n, k, i}+s_{n, k, i} \phi_{n, k, i}, \\
& \tau_{n, k, i+1}=\tau_{n, k, i}+s_{n, k, i} \leq \tau_{n, k}+\Delta \tau_{n}=\tau_{n, k+1} .
\end{aligned}
$$

Note that with this definition

$$
\left\|v_{n, k, i+1}-v_{n, k, i}\right\|=\tau_{n, k, i+1}-\tau_{n, k, i}=s_{n, k, i} .
$$


Next, let us define

$$
v_{n, k+1}=\lim _{i} v_{n, k, i}=v_{n, k}+\sum_{i=0}^{\infty}\left(v_{n, k, i+1}-v_{n, k, i}\right) \quad \text { and } \quad \bar{\tau}_{n, k}=\lim _{i} \tau_{n, k, i}=\tau_{n, k}+\sum_{i=0}^{\infty} s_{n, k, i} .
$$

Note that the sequence $\tau_{n, k, i}$ is non-decreasing, with respect to $i$, and bounded from above by $\tau_{n, k+1}=\tau_{n, k}+\Delta \tau_{n}$, thus the limit $\bar{\tau}_{n, k}$ exists and is bounded by the same constant. Moreover, the limit of $v_{n, k, i}$ exists because

$$
\sum_{i=0}^{\infty}\left\|v_{n, k, i+1}-v_{n, k, i}\right\|=\sum_{i=0}^{\infty} s_{n, k, i}=\bar{\tau}_{n, k}-\tau_{n, k} .
$$

In particular $\left\|v_{n, k+1}-v_{n, k}\right\| \leq \Delta \tau_{n}$. Finally, let

$$
t_{n, k+1}=t_{n, k}+\left(\tau_{n, k+1}-\bar{\tau}_{n, k}\right) .
$$

As in the previous section, let $\bar{k}_{n}=\sup \left\{k: t_{n, k}<t_{1}\right\}$ and denote $\mathcal{T}_{n}=\bar{k}_{n} \Delta \tau_{n} \geq\left(t_{1}-t_{0}\right)$. Now we define the sequences $v_{n}:\left[0, \mathcal{T}_{n}\right) \rightarrow \mathcal{V}$ and $t_{n}:\left[0, \mathcal{T}_{n}\right) \rightarrow\left[t_{0}, t_{1}\right]$. In the subinterval $\left[\tau_{n, k}, \bar{\tau}_{n, k}\right)$ we define $v_{n}$ to be the piecewise affine interpolation of $v_{n, k, i}$ in the points $\tau_{n, k, i}$ while in the subinterval $\left[\bar{\tau}_{n, k}, \tau_{n, k+1}\right]$ we set $v_{n}=v_{n, k+1}$. The definition of $t_{n}$ is somehow complementary: in the subinterval $\left[\tau_{n, k}, \bar{\tau}_{n, k}\right)$ we define $t_{n}=t_{n, k}$ while in the subinterval $\left[\bar{\tau}_{n, k}, \tau_{n, k+1}\right]$ we take the affine interpolation of $t_{n, k}$ and $t_{n, k+1}$. In this way in the subinterval $\left[\tau_{n, k}, \bar{\tau}_{n, k}\right)$ we have $\left\|v_{n}^{\prime}\right\|=1$, thanks to (5.4), and $t_{n}^{\prime}=0$; in the subinterval $\left[\bar{\tau}_{n, k}, \tau_{n, k+1}\right]$ we have $v_{n}^{\prime}=0$ and $t_{n}^{\prime}=1$, thanks to (5.5). Therefore we still have $t_{n}^{\prime}+\left\|v_{n}^{\prime}\right\|=1$.

Proposition 5.1. If $t_{n, k+1}>t_{n, k}$ then $v_{n, k+1}$ satisfies the equilibrium condition

$$
\left\|\partial_{v} \mathcal{F}\left(t_{n, k}, v_{n, k+1}\right)\right\|=0 .
$$

Proof. If $t_{n, k+1}>t_{n, k}$ then $0 \leq \bar{\tau}_{n, k}<\tau_{n, k+1}$ and $s_{n, k, i}<\Delta \tau_{n}-\left(\tau_{n, k, i}-\tau_{n, k}\right)$ for every $i$, hence by $(5.3)$

$$
\partial_{v} \mathcal{F}\left(t_{n, k}, v_{n, k, i+1}\right)\left[\phi_{n, k, i}\right]=0 .
$$

By (5.1) and by the uniform continuity $(2.6)$ of $\partial_{v} \mathcal{F}$ it follows that

$$
\left\|\partial_{v} \mathcal{F}\left(t_{n, k}, v_{n, k, i}\right)\right\|=\left|\partial_{v} \mathcal{F}\left(t_{n, k}, v_{n, k, i}\right)\left[\phi_{n, k, i}\right]\right| \leq \mathcal{C}\left(\left\|v_{n, k, i+1}-v_{n, k, i}\right\|\right)=\mathcal{C}\left(s_{n, k, i}\right) .
$$

Then, by the lower semi-continuity (2.4) of $\left\|\partial_{v} \mathcal{F}\right\|$ and by the convergence of $v_{n, k, i}$ we get

$$
\left\|\partial_{v} \mathcal{F}\left(t_{n, k}, v_{n, k+1}\right)\right\| \leq \liminf _{i}\left\|\partial_{v} \mathcal{F}\left(t_{n, k}, v_{n, k, i}\right)\right\| \leq \liminf _{i} \mathcal{C}\left(s_{n, k, i}\right)=0,
$$

which is (5.6).

Proposition 5.2. The following incremental energy estimate holds

$$
\begin{aligned}
\mathcal{F}\left(t_{n, k+1}, v_{n, k+1}\right) \leq & \mathcal{F}\left(t_{n, k}, v_{n, k}\right)-\int_{\tau_{n, k}}^{\tau_{n, k+1}}\left\|\partial_{v} \mathcal{F}\left(t_{n, k}, v_{n}(\tau)\right)\right\| \mathrm{d} \tau \\
& +\int_{\tau_{n, k}}^{\tau_{n, k+1}} \partial_{t} \mathcal{F}\left(t_{n}(\tau), v_{n}(\tau)\right) t_{n}^{\prime}(\tau) \mathrm{d} \tau+3 \mathcal{C}\left(\Delta \tau_{n}\right) \Delta \tau_{n},
\end{aligned}
$$

where $\mathcal{C}$ is the modulus of continuity appearing in (2.6). 
Proof. Arguing as in the proof of Proposition 4.2 we obtain

$$
\begin{aligned}
\mathcal{F}\left(t_{n, k+1}, v_{n, k+1}\right)= & \mathcal{F}\left(t_{n, k}, v_{n, k+1}\right)+\int_{t_{n, k}}^{t_{n, k+1}} \partial_{t} \mathcal{F}\left(t, v_{n, k+1}\right) \mathrm{d} t \\
= & \mathcal{F}\left(t_{n, k}, v_{n, k}\right)+\int_{\tau_{n, k}}^{\tau_{n, k+1}} \partial_{v} \mathcal{F}\left(t_{n, k}, v_{n}(\tau)\right)\left[v_{n}^{\prime}(\tau)\right] \mathrm{d} \tau \\
& +\int_{\tau_{n, k}}^{\tau_{n, k+1}} \partial_{t} \mathcal{F}\left(t_{n}(\tau), v_{n, k+1}\right) t_{n}^{\prime}(\tau) \mathrm{d} \tau
\end{aligned}
$$

but this time we cannot use minimality for $v_{n, k+1}$. Thus, for $\tau \in\left[\tau_{n, k, i}, \tau_{n, k, i+1}\right]$ we have $v_{n}^{\prime}(\tau)=\phi_{n, k, i} \in$ $\operatorname{argmin}\left\{\partial_{v} \mathcal{F}\left(t_{n, k}, v_{n, k, i}\right)[\phi]:\|\phi\|=1\right\}$ and then by (2.6) we can write

$$
\begin{aligned}
\partial_{v} \mathcal{F}\left(t_{n, k}, v_{n}(\tau)\right)\left[v_{n}^{\prime}(\tau)\right] & \leq \partial_{v} \mathcal{F}\left(t_{n, k}, v_{n, k, i}\right)\left[\phi_{n, k, i}\right]+\mathcal{C}\left(\left\|v_{n, k, i+1}-v_{n}(\tau)\right\|\right) \\
& \leq-\left\|\partial_{v} \mathcal{F}\left(t_{n, k}, v_{n, k, i}\right)\right\|+\mathcal{C}\left(s_{n, k, i}\right) \\
& \leq-\left\|\partial_{v} \mathcal{F}\left(t_{n, k}, v_{n}(\tau)\right)\right\|+2 \mathcal{C}\left(s_{n, k, i}\right) .
\end{aligned}
$$

Then

$$
\begin{aligned}
\int_{\tau_{n, k, i}}^{\tau_{n, k, i+1}} \partial_{v} \mathcal{F}\left(t_{n, k}, v_{n}(\tau)\right)\left[v_{n}^{\prime}(\tau)\right] \mathrm{d} \tau \leq & -\int_{\tau_{n, k, i}}^{\tau_{n, k, i+1}}\left\|\partial_{v} \mathcal{F}\left(t_{n, k}, v_{n}(\tau)\right)\right\| \mathrm{d} \tau \\
& +2 \mathcal{C}\left(s_{n, k, i}\right)\left|\tau_{n, k, i+1}-\tau_{n, k, i}\right|
\end{aligned}
$$

and hence in the subinterval $\left[\tau_{n, k}, \bar{\tau}_{n, k}\right]$ we have

$$
\begin{aligned}
\int_{\tau_{n, k}}^{\bar{\tau}_{n, k}} \partial_{v} \mathcal{F}\left(t_{n, k}, v_{n}(\tau)\right)\left[v_{n}^{\prime}(\tau)\right] \mathrm{d} \tau \leq & -\int_{\tau_{n, k}}^{\bar{\tau}_{n, k}}\left\|\partial_{v} \mathcal{F}\left(t_{n, k}, v_{n}(\tau)\right)\right\| \mathrm{d} \tau \\
& +2 \mathcal{C}\left(\Delta \tau_{n}\right)\left|\bar{\tau}_{n, k}-\tau_{n, k}\right| .
\end{aligned}
$$

In the subinterval $\left[\bar{\tau}_{n, k}, \tau_{n, k+1}\right]$ (if it is not a single point) we have by Proposition 5.1

$$
\partial_{v} \mathcal{F}\left(t_{n, k}, v_{n}(\tau)\right)\left[v_{n}^{\prime}(\tau)\right]=\left\|\partial_{v} \mathcal{F}\left(t_{n, k}, v_{n, k+1}\right)\right\|=0 .
$$

Therefore in the whole interval $\left[\tau_{n, k}, \tau_{n, k+1}\right]$ we can write

$$
\int_{\tau_{n, k}}^{\tau_{n, k+1}} \partial_{v} \mathcal{F}\left(t_{n, k}, v_{n}(\tau)\right)\left[v_{n}^{\prime}(\tau)\right] \mathrm{d} \tau \leq-\int_{\tau_{n, k}}^{\tau_{n, k+1}}\left\|\partial_{v} \mathcal{F}\left(t_{n, k}, v_{n}(\tau)\right)\right\| \mathrm{d} \tau+2 \mathcal{C}\left(\Delta \tau_{n}\right) \Delta \tau_{n} .
$$

Finally, again by $(2.6)$

$$
\int_{\tau_{n, k}}^{\tau_{n, k+1}} \partial_{t} \mathcal{F}\left(t_{n}(\tau), v_{n, k+1}\right) t_{n}^{\prime}(\tau) \mathrm{d} \tau \leq \int_{\tau_{n, k}}^{\tau_{n, k+1}} \partial_{t} \mathcal{F}\left(t_{n}(\tau), v_{n}(\tau)\right) t_{n}^{\prime}(\tau) \mathrm{d} \tau+\mathcal{C}\left(\Delta \tau_{n}\right) \Delta \tau_{n},
$$

which concludes the proof.

\subsection{Compactness and convergence}

Arguing exactly as in Section 4.2 we can prove the next results; this is possible thanks to the fact that the proofs of Proposition 4.3, Theorem 4.4 and Corollary 4.5 depend only on Propositions 4.1 and 4.2.

Proposition 5.3. Let $\left(t_{n}, v_{n}\right):\left[0, \mathcal{T}_{n}\right) \rightarrow\left[t_{0}, t_{1}\right] \times \mathcal{V}$ be given by Section 5.1. Let $0<\mathcal{T}<\liminf { }_{n} \mathcal{T}_{n}$. There exists a subsequence (not relabelled) such that $t_{n} \stackrel{*}{\rightarrow} t$ in $W^{1, \infty}(0, \mathcal{T})$ and $v_{n} \stackrel{*}{\rightarrow} v$ in $W^{1, \infty}(0, \mathcal{T} ; \mathcal{V})$. In particular $t_{n}\left(\tau_{n}\right) \rightarrow t(\tau)$ and $v_{n}\left(\tau_{n}\right) \rightarrow v(\tau)$ in $\mathcal{V}$ if $\tau_{n} \rightarrow \tau$. Moreover $0 \leq t^{\prime} \leq 1$ and $\left\|v^{\prime}\right\| \leq 1$ a.e. in $(0, \mathcal{T})$. 
Theorem 5.4. Let $\left(t_{n}, v_{n}\right)$ and $(t, v)$ be as in Proposition 5.3; then $(t, v)$ is (a parametrization of) a quasi-static evolution, i.e. it satisfies $(t(0), v(0))=\left(t_{0}, v_{0}\right), 0 \leq t^{\prime} \leq 1,\left\|v^{\prime}\right\| \leq 1$, and

$\left(S^{\prime}\right)$ for every $\tau>0$ with $t^{\prime}(\tau)>0$ it holds

$$
\left\|\partial_{v} \mathcal{F}(t(\tau), v(\tau))\right\|=0
$$

$\left(E^{\prime}\right)$ for every $\tau$ it holds

$$
\mathcal{F}(t(\tau), v(\tau))=\mathcal{F}\left(t_{0}, v_{0}\right)-\int_{0}^{\tau}\left\|\partial_{v} \mathcal{F}(t(s), v(s))\right\| \mathrm{d} s+\int_{0}^{\tau} \partial_{t} \mathcal{F}(t(s), v(s)) t^{\prime}(s) \mathrm{d} s .
$$

Corollary 5.5. $\mathcal{F}\left(t_{n}(\tau), v_{n}(\tau)\right) \rightarrow \mathcal{F}(t(\tau), v(\tau))$ for every $\tau \in[0, \mathcal{T})$.

\section{Proof of the approximation RESUlt}

In this section we will prove Theorem 2.3. Let $\left(t_{h}, v_{h}\right):[0, \mathcal{T}) \rightarrow\left[t_{0}, t_{1}\right] \times \mathcal{V}_{h} \subset \mathcal{V}$ be a family of Lipschitz maps with $\left(t_{h}(0), v_{h}(0)\right)=\left(t_{0}, v_{h, 0}\right), 0 \leq t_{h}^{\prime} \leq 1$ and $\left\|v_{h}^{\prime}\right\| \leq 1$, and such that

$\left(S_{h}^{\prime}\right)$ for every $\tau$ with $t_{h}^{\prime}(\tau)>0$ it holds

$$
\left\|\partial_{v} \mathcal{F}_{h}\left(t_{h}(\tau), v_{h}(\tau)\right)\right\|_{h}=0
$$

$\left(E_{h}^{\prime}\right)$ for every $\tau$ it holds

$$
\mathcal{F}_{h}\left(t_{h}(\tau), v_{h}(\tau)\right)=\mathcal{F}_{h}\left(t_{0}, v_{0}\right)-\int_{0}^{\tau}\left\|\partial_{v} \mathcal{F}_{h}\left(t_{h}(s), v_{h}(s)\right)\right\|_{h} \mathrm{~d} s+\int_{0}^{\tau} \partial_{t} \mathcal{F}_{h}\left(t_{h}(s), v_{h}(s)\right) t_{h}^{\prime}(s) \mathrm{d} s .
$$

By assumption $\left(t_{h}, v_{h}\right)$ is sequentially weakly* compact in $W^{1, \infty}(0, \mathcal{T}) \times W^{1, \infty}(0, \mathcal{T} ; \mathcal{V})$, therefore by the arguments of Proposition 4.3 there exists a subsequence (not relabelled) such that $t_{h} \stackrel{*}{\rightarrow} t$ in $W^{1, \infty}(0, \mathcal{T}$ ) and $v_{h} \stackrel{*}{\rightarrow} v$ in $W^{1, \infty}(0, \mathcal{T} ; \mathcal{V}), t_{h}\left(\tau_{h}\right) \rightarrow t(\tau)$ and $v_{h}\left(\tau_{h}\right) \rightarrow v(\tau)$ if $\tau_{h} \rightarrow \tau, 0 \leq t^{\prime} \leq 1$ and $\left\|v^{\prime}\right\| \leq 1$ a.e. in $(0, \mathcal{T})$. In particular $t_{h}(\tau) \rightarrow t(\tau), v_{h}(\tau) \rightarrow v(\tau)$ for every $\tau \in[0, \mathcal{T})$.

Now, to check that the limit $(t, v)$ satisfies conditions $\left(S^{\prime}\right)$ and $\left(E^{\prime}\right)$ we follow the same arguments used in the proof of Theorem 4.4, roughly speaking replacing $\left(t_{n}, v_{n}\right)$ with $\left(t_{h}, v_{h}\right)$ and conditions $(2.3)-(2.5)$ with $(2.7)-(2.9)$.

Let $\tau$ with $t^{\prime}(\tau)>0$. Since $t_{h}$ converges to $t$ pointwise there exists a sequence $\tau_{h} \rightarrow \tau$ such that $t_{h}^{\prime}\left(\tau_{h}\right)>0$ and thus $\left\|\partial_{v} \mathcal{F}_{h}\left(t_{h}\left(\tau_{h}\right), v\left(\tau_{h}\right)\right)\right\|_{h}=0$, by $\left(S_{h}^{\prime}\right)$. As $\tau_{h} \rightarrow \tau$ we also have $v_{h}\left(\tau_{h}\right) \rightarrow v(\tau)$ in $\mathcal{V}$. Therefore, thanks to $(2.8)$ we get

$$
\left\|\partial_{v} \mathcal{F}(t(\tau), v(\tau))\right\| \leq \liminf _{h}\left\|\partial_{v} \mathcal{F}\left(t_{h}\left(\tau_{h}\right), v\left(\tau_{h}\right)\right)\right\|_{h}=0 .
$$

It remains to show that $\left(E^{\prime}\right)$ follows from $\left(E_{h}^{\prime}\right)$. Taking the limsup in (6.2) yields

$$
\begin{aligned}
\limsup _{h} \mathcal{F}_{h}\left(t_{h}(\tau), v_{h}(\tau)\right) \leq & \lim _{h} \mathcal{F}_{h}\left(t_{0}, v_{h, 0}\right)-\liminf _{h} \int_{0}^{\tau}\left\|\partial_{v} \mathcal{F}_{h}\left(t_{h}(s), v_{h}(s)\right)\right\|_{h} \mathrm{~d} s \\
& +\underset{h}{\limsup } \int_{0}^{\tau} \partial_{t} \mathcal{F}_{h}\left(t_{h}(s), v_{h}(s)\right) t_{h}^{\prime}(s) \mathrm{d} s .
\end{aligned}
$$

By (2.10) we know that

$$
\lim _{h} \mathcal{F}_{h}\left(t_{0}, v_{h, 0}\right)=\mathcal{F}\left(t_{0}, v_{0}\right) .
$$

As $t_{h}(s) \rightarrow t(s)$ and $v_{h}(s) \rightarrow v(s)$ by $(2.8)$ we have $\left\|\partial_{v} \mathcal{F}(t(s), v(s))\right\| \leq \liminf _{h}\left\|\partial_{v} \mathcal{F}_{h}\left(t_{h}(s), v_{h}(s)\right)\right\|_{h}$ and then by Fatou's Lemma

$$
\int_{0}^{\tau}\left\|\partial_{v} \mathcal{F}(t(s), v(s))\right\| \mathrm{d} s \leq \liminf _{h} \int_{0}^{\tau}\left\|\partial_{v} \mathcal{F}_{h}\left(t_{h}(s), v_{h}(s)\right)\right\|_{h} \mathrm{~d} s .
$$


By (2.9) we have $\partial_{t} \mathcal{F}(t(s), v(s))=\lim _{h} \partial_{t} \mathcal{F}_{h}\left(t_{h}(s), v_{h}(s)\right) \leq C$ where (by assumption) the upper bound $C$ is uniform. By dominated convergence it follows that $\partial_{t} \mathcal{F}_{h}\left(t_{h}(\cdot), v_{h}(\cdot)\right) \rightarrow \partial_{t} \mathcal{F}(t(\cdot), v(\cdot))$ strongly in $L^{1}(0, \mathcal{T})$. Since $t_{h}^{\prime} \stackrel{*}{\rightarrow} t^{\prime}$ in $L^{\infty}(0, \mathcal{T})$ we get

$$
\lim _{h} \int_{0}^{\tau} \partial_{t} \mathcal{F}_{h}\left(t_{h}(s), v_{h}(s)\right) t_{h}^{\prime}(s) \mathrm{d} s=\int_{0}^{\tau} \partial_{t} \mathcal{F}(t(s), v(s)) t^{\prime}(s) \mathrm{d} s .
$$

In conclusion,

$$
\underset{h}{\limsup } \mathcal{F}_{h}\left(t_{h}(\tau), v_{h}(\tau)\right) \leq \mathcal{F}\left(t_{0}, v_{0}\right)-\int_{0}^{\tau}\left\|\partial_{v} \mathcal{F}(t(s), v(s))\right\| \mathrm{d} s+\int_{0}^{\tau} \partial_{t} \mathcal{F}(t(s), v(s)) t^{\prime}(s) \mathrm{d} s .
$$

Since $\left\|v^{\prime}\right\| \leq 1$ and since $\tau \mapsto \mathcal{F}(t(\tau), v(\tau))$ is absolutely continuous by the chain rule we deduce again that

$$
\mathcal{F}\left(t_{0}, v_{0}\right)-\int_{0}^{\tau}\left\|\partial_{v} \mathcal{F}(t(s), v(s))\right\| \mathrm{d} s+\int_{0}^{\tau} \partial_{t} \mathcal{F}(t(s), v(s)) t^{\prime}(s) \mathrm{d} s \leq \mathcal{F}(t(\tau), v(\tau))
$$

and thus $\lim \sup _{h} \mathcal{F}_{h}\left(t_{h}(\tau), v_{h}(\tau)\right) \leq \mathcal{F}(t(\tau), v(\tau))$. The liminf inequality

$$
\mathcal{F}(t(\tau), v(\tau)) \leq \liminf _{h} \mathcal{F}_{h}\left(t_{h}(\tau), v_{h}(\tau)\right)
$$

is provided by $(2.7)$. Therefore

$$
\limsup _{h} \mathcal{F}_{h}\left(t_{h}(\tau), v_{h}(\tau)\right)=\lim _{h} \mathcal{F}_{h}\left(t_{h}(\tau), v_{h}(\tau)\right)=\mathcal{F}(t(\tau), v(\tau)) .
$$

As a consequence from (6.3) we get $\left(E^{\prime}\right)$. Note also that, in the language of $\Gamma$-convergence, $\left(t_{h}(\tau), v_{h}(\tau)\right)$ is a recovery sequence for every $\tau \in[0, \mathcal{T})$.

\section{EXISTENCE IN A WEAKER NORM}

In this section we will prove the existence result stated in Theorem 2.5. We will not follow the proofs of the previous existence Theorems; we will use instead a "Galerkin proof" approximating the evolution in finite dimensional spaces. To this end, let $\mathcal{V}_{h}$ be a monotone sequence of finite dimensional subspaces of $\mathcal{V}$ with $\cup_{h} \mathcal{V}_{h}$ dense in $\mathcal{V}$. Let $\mathcal{F}_{h}$ be the restriction to $\mathcal{V}_{h}$ of the energy functional $\mathcal{F}$. Being $\mathcal{V}_{h} \subset \mathcal{V} \subset \mathcal{W}$ and being $\mathcal{V}_{h}$ finite dimensional, the norms $\|\cdot\|_{\mathcal{V}}$ and $\|\cdot\|_{\mathcal{W}}$ are equivalent in $\mathcal{V}_{h}$ as it is for the weak and strong topologies.

First of all let us prove the existence of the discrete evolutions. As $\mathcal{F}_{h}=\mathcal{F}$ in $\mathcal{V}_{h}$ it turns out that the energy functionals $\mathcal{F}_{h}:\left[t_{0}, t_{1}\right] \times \mathcal{V}_{h} \rightarrow[0,+\infty)$ are of class $C^{1}$ with respect to $\|\cdot\|_{\mathcal{V}}$ and thus with respect to $\|\cdot\|_{\mathcal{W}}$. For $v \in \mathcal{V}_{h}$ denote by $\nabla_{\mathcal{V}} \mathcal{F}_{h}(t, v)$ and by $\nabla_{\mathcal{W}} \mathcal{F}_{h}(t, v)$ the gradients in the first and second norm respectively. For $v \in \mathcal{V}_{h}$

$$
\left\|\nabla_{\mathcal{W}} \mathcal{F}_{h}(t, v)\right\|=\max \left\{\partial_{v} \mathcal{F}_{h}(t, v)[\phi]: \phi \in \mathcal{V}_{h},\|\phi\|_{\mathcal{W}} \leq 1\right\}
$$

and similarly for $\left\|\nabla_{\mathcal{V}} \mathcal{F}_{h}(t, v)\right\|$. Since $\mathcal{F}_{h}$ is of class $C^{1}$ in $\left[t_{0}, t_{1}\right] \times \mathcal{V}_{h}$ it is clear that

$$
\begin{gathered}
\mathcal{F}_{h}(t, v) \leq \liminf _{m} \mathcal{F}_{h}\left(t_{m}, v_{m}\right) \quad \text { for } t_{m} \rightarrow t \text { and } v_{m} \rightarrow v, \\
\left\|\nabla_{\mathcal{W}} \mathcal{F}_{h}(t, v)\right\| \leq \liminf _{m}\left\|\nabla_{\mathcal{W}} \mathcal{F}_{h}\left(t_{m}, v_{m}\right)\right\| \quad \text { for } t_{m} \rightarrow t \text { and } v_{m} \rightarrow v, \\
\partial_{t} \mathcal{F}_{h}(t, v)=\lim _{m} \partial_{t} \mathcal{F}_{h}\left(t_{m}, v_{m}\right) \quad \text { for } t_{m} \rightarrow t \text { and } v_{m} \rightarrow v .
\end{gathered}
$$

Remember that in the finite dimensional setting weak and strong convergence coincide. Now, let us check that there exists a modulus of continuity $\mathcal{C}_{h}$ such that in $\left[t_{0}, t_{1}\right] \times \mathcal{V}_{h}$ we have

$$
\left\|\nabla_{\mathcal{W}} \mathcal{F}_{h}(t, v)-\nabla_{\mathcal{W}} \mathcal{F}_{h}(t, w)\right\|+\left|\partial_{t} \mathcal{F}_{h}(t, v)-\partial_{t} \mathcal{F}_{h}(t, w)\right| \leq \mathcal{C}_{h}\left(\|v-w\|_{\mathcal{W}}\right)
$$


Note that, being $\mathcal{F}_{h}=\mathcal{F}$ on $\mathcal{V}_{h}$, for $v \in \mathcal{V}_{h}$ we have

$$
\left\|\partial_{v} \mathcal{F}(t, v)\right\|=\max \left\{\partial_{v} \mathcal{F}(t, v)[\phi]: \phi \in \mathcal{V},\|\phi\|_{\mathcal{V}} \leq 1\right\} \geq\left\|\nabla_{\mathcal{V}} \mathcal{F}_{h}(t, v)\right\|,
$$

(note that the last term is the norm of the finite dimensional functional $\mathcal{F}_{h}$ ) and then, by equivalence of norms, we can write

$$
C_{h}\left\|\nabla_{\mathcal{W}} \mathcal{F}_{h}(t, v)\right\| \leq\left\|\nabla_{\mathcal{V}} \mathcal{F}_{h}(t, v)\right\| \leq\left\|\partial_{v} \mathcal{F}(t, v)\right\| .
$$

Therefore, again by the equivalence of norms, from

$$
\left\|\partial_{v} \mathcal{F}(t, v)-\partial_{v} \mathcal{F}(t, w)\right\|+\left|\partial_{t} \mathcal{F}(t, v)-\partial_{t} \mathcal{F}(t, w)\right| \leq \mathcal{C}\left(\|v-w\|_{\mathcal{V}}\right)
$$

we get (7.4). Now, let $v_{h, 0} \in \mathcal{V}_{h}$ with $v_{h, 0} \rightarrow v_{0}$ in $\mathcal{V}$. Thanks to (7.1)-(7.4) we can invoke Theorem 2.2 which provides the existence of a quasi-static evolution $\left(t_{h}, v_{h}\right):\left[0, \mathcal{I}_{h}\right) \rightarrow\left[t_{0}, t_{1}\right] \times \mathcal{V}_{h}$ with $\left(t_{h}(0), v_{h}(0)\right)=\left(t_{0}, v_{h, 0}\right)$, $0 \leq t_{h}^{\prime} \leq 1$ and $\left\|v_{h}^{\prime}\right\|_{\mathcal{W}} \leq 1$ and such that

$\left(S_{h}^{\prime}\right)$ for every $\tau$ with $t_{h}^{\prime}(\tau)>0$

$$
\left\|\nabla_{\mathcal{W}} \mathcal{F}_{h}\left(t_{h}(\tau), v_{h}(\tau)\right)\right\|=0
$$

$\left(E_{h}^{\prime}\right)$ for every $\tau$

$$
\begin{aligned}
\mathcal{F}_{h}\left(t_{h}(\tau), v_{h}(\tau)\right)= & \mathcal{F}_{h}\left(t_{0}, v_{h, 0}\right)-\int_{0}^{\tau}\left\|\nabla_{\mathcal{W}} \mathcal{F}_{h}\left(t_{h}(s), v_{h}(s)\right)\right\| \mathrm{d} s \\
& +\int_{0}^{\tau} \partial_{t} \mathcal{F}_{h}\left(t_{h}(s), v_{h}(s)\right) t_{h}^{\prime}(s) \mathrm{d} s .
\end{aligned}
$$

Next, let us prove compactness. As already observed in Proposition 4.3, we have $\mathcal{T}_{h} \geq\left(t_{1}-t_{0}\right)$. Let $0<\mathcal{T}<$ $\lim \inf _{h} \mathcal{T}_{h}$. Since $t_{h}$ is bounded in $W^{1, \infty}(0, \mathcal{T})$ and $v_{h}$ is bounded in $W^{1, \infty}(0, \mathcal{T} ; \mathcal{W})$ it follows (by the arguments of Prop. 4.3) that there exists a subsequence (not relabelled) such that $t_{h} \stackrel{*}{\rightarrow} t$ in $W^{1, \infty}(0, \mathcal{T})$ and $v_{h} \stackrel{*}{\rightarrow} v$ in $W^{1, \infty}(0, \mathcal{T} ; \mathcal{W})$. Moreover, for every $\tau \in[0, \mathcal{T})$ we have $t_{h}(\tau) \rightarrow t(\tau)$ and $v_{h}(\tau) \rightarrow v(\tau)$ in $\mathcal{W}$. Clearly $0 \leq t^{\prime} \leq 1$ and $\left\|v^{\prime}\right\|_{\mathcal{W}} \leq 1$ a.e. in $(0, \mathcal{T})$.

Now, let us see that $v_{h}(\tau) \rightarrow v(\tau)$ in the weak topology of $\mathcal{V}$ (we will need this property in the sequel). By the chain rule, for every $\tau_{1} \leq \tau_{2}$ we can write

$$
\mathcal{F}_{h}\left(t_{h}\left(\tau_{2}\right), v_{h}\left(\tau_{2}\right)\right)=\mathcal{F}_{h}\left(t_{h}\left(\tau_{1}\right), v_{h}\left(\tau_{1}\right)\right)+\int_{\tau_{1}}^{\tau_{2}} \mathcal{F}_{h}^{\prime}\left(t_{h}(\tau), v_{h}(\tau)\right) \mathrm{d} \tau
$$

where $\mathcal{F}_{h}^{\prime}$ denotes the (total) derivative with respect to $\tau$. At the same time by $\left(E_{h}^{\prime}\right)$ we have

$$
\mathcal{F}_{h}\left(t_{h}\left(\tau_{2}\right), v_{h}\left(\tau_{2}\right)\right) \leq \mathcal{F}_{h}\left(t_{h}\left(\tau_{1}\right), v_{h}\left(\tau_{1}\right)\right)+\int_{\tau_{1}}^{\tau_{2}} \partial_{t} \mathcal{F}_{h}\left(t_{h}(\tau), v_{h}(\tau)\right) t_{h}^{\prime}(\tau) \mathrm{d} \tau
$$

Since $t_{h}^{\prime} \leq 1$ and by (2.17) it follows that for a.e. $\tau$ it holds

$$
\mathcal{F}_{h}^{\prime}\left(t_{h}(\tau), v_{h}(\tau)\right) \leq \partial_{t} \mathcal{F}_{h}\left(t_{h}(\tau), v_{h}(\tau)\right) t_{h}^{\prime}(\tau) \leq A \mathcal{F}_{h}\left(t_{h}(\tau), v_{h}(\tau)\right)+B
$$

(for $A, B$ independent of $h$ ). By Gronwall Lemma

$$
\mathcal{F}\left(t_{h}(\tau), v_{h}(\tau)\right)=\mathcal{F}_{h}\left(t_{h}(\tau), v_{h}(\tau)\right) \leq C\left(\mathcal{F}_{h}\left(t_{0}, v_{h, 0}\right)+1\right) e^{A \tau} \leq C^{\prime}
$$

and hence $\mathcal{F}_{h}\left(t_{h}(\tau), v_{h}(\tau)\right)$ is bounded, uniformly with respect to $\tau \in[0, \mathcal{T})$ and $h \in \mathbb{N}$. By the coercivity of $\mathcal{F}(t, \cdot)$ it follows that $\left\|v_{h}(\tau)\right\|_{\mathcal{V}}$ is bounded. We already know that for every $\tau$ we have $v_{h}(\tau) \rightarrow v(\tau)$ in the weak topology of $\mathcal{W}$. Since $v_{h}(\tau)$ is bounded in $\mathcal{V}$, which is reflexive, for every subsequence $v_{h_{k}}(\tau)$ there exists a further subsequence (not relabelled) such that $v_{h_{k}}(\tau) \rightarrow z$ in the weak topology of $\mathcal{V}$. Since $\mathcal{V}$ is continuously 
embedded in $\mathcal{W}$ it follows that $v_{h_{k}}(\tau) \rightarrow z$ in the weak topology of $\mathcal{W}$ and thus $z=v(\tau)$. As a consequence for the whole sequence we have $v_{h}(\tau) \rightarrow v(\tau)$ in $\mathcal{V}$.

Finally, let us see the convergence of $\left(S_{h}^{\prime}\right)$ and $\left(E_{h}^{\prime}\right)$. In order to pass to the limit in (7.5) it is sufficient to show that for every $\tau$

$$
\left\|\nabla_{\mathcal{W}} \mathcal{F}(t(\tau), v(\tau))\right\| \leq \liminf _{h}\left\|\nabla_{\mathcal{W}} \mathcal{F}_{h}\left(t_{h}(\tau), v_{h}(\tau)\right)\right\|
$$

For $h \geq h^{\prime}$ and $\phi_{h^{\prime}} \in \mathcal{V}_{h^{\prime}}$ with $\left\|\phi_{h^{\prime}}\right\|_{\mathcal{W}} \leq 1$ we can write

$$
\begin{aligned}
\left\|\nabla_{\mathcal{W}} \mathcal{F}_{h}\left(t_{h}(\tau), v_{h}(\tau)\right)\right\| & =\max \left\{\partial_{v} \mathcal{F}\left(t_{h}(\tau), v_{h}(\tau)\right)[\phi]: \phi \in \mathcal{V}_{h},\|\phi\|_{\mathcal{W}} \leq 1\right\} \\
& \geq \partial_{v} \mathcal{F}\left(t_{h}(\tau), v_{h}(\tau)\right)\left[\phi_{h^{\prime}}\right]
\end{aligned}
$$

Since $v_{h}(\tau) \rightarrow v(\tau)$ in $\mathcal{V}$ we can use (2.14) to get

$$
\liminf _{h}\left\|\nabla_{\mathcal{W}} \mathcal{F}_{h}\left(t_{h}(\tau), v_{h}(\tau)\right)\right\| \geq \partial_{v} \mathcal{F}(t(\tau), v(\tau))\left[\phi_{h^{\prime}}\right] \quad \text { for every } \phi_{h^{\prime}} \in \mathcal{V}_{h^{\prime}}
$$

Since $\cup_{h^{\prime}} \mathcal{V}_{h^{\prime}}$ is dense in $\mathcal{V}$ it is enough to take the supremum with respect to $\phi_{h^{\prime}} \in \mathcal{V}_{h^{\prime}}$ with $\left\|\phi_{h^{\prime}}\right\|_{\mathcal{W}} \leq$ 1. Note that $\partial_{v} \mathcal{F}\left(t_{h}(\tau), v_{h}(\tau)\right)\left[\phi_{h^{\prime}}\right]=\partial_{v} \mathcal{F}_{h}\left(t_{h}(\tau), v_{h}(\tau)\right)\left[\phi_{h^{\prime}}\right]$ is measurable, therefore its pointwise limit $\partial_{v} \mathcal{F}(t(\tau), v(\tau))\left[\phi_{h^{\prime}}\right]$ and then the supremum $\left\|\nabla_{\mathcal{W}} \mathcal{F}(t(\tau), v(\tau))\right\|$ are measurable, and actually integrable.

Following the arguments of Section 6, to pass to the limit in (7.6) it is sufficient to show that

$$
\begin{array}{r}
\mathcal{F}(t(\tau), v(\tau)) \leq \liminf _{h} \mathcal{F}_{h}\left(t_{h}(\tau), v_{h}(\tau)\right), \\
\limsup \mathcal{F}_{h}\left(t_{0}, v_{h, 0}\right) \leq \mathcal{F}\left(t_{0}, v_{0}\right), \\
\lim _{h} \partial_{t} \mathcal{F}_{h}\left(t_{h}(\cdot), v_{h}(\cdot)\right)=\partial_{t} \mathcal{F}(t(\cdot), v(\cdot)) \text { in } L^{1}(0, T) .
\end{array}
$$

Remembering that $\mathcal{F}_{h}$ is just the restriction of $\mathcal{F}$ on $\mathcal{V}_{h}$ the first and the third condition follow respectively from (2.13) and (2.15) together with the uniform bound (7.7). The second is instead a direct consequence of the fact that $v_{h, 0} \rightarrow v_{0}$ strongly in $\mathcal{V}$ and thus the initial datum is well prepared.

\section{A PHASE FIELD MODEL FOR BRITTLE FRACTURE}

After [18] fracture propagation has been extensively studied in the framework of quasi-static rate-independent evolutions, following various approaches. At the current stage, what makes the biggest difference in the mathematical and mechanical aspects is the representation of the crack: on the one hand is the representation by a set, on the other hand the representation by means of a phase-field variable.

In the former case, $B V$ or parametrized solutions are well defined only along regular pre-assigned crack paths $[20,31,38]$. For general unknown cracks, $B V$ solutions are instead not applicable since in general the energy is not differentiable: a couple of interesting works indicate indeed a delicate behaviour of the slope in the $L^{2}$-norm of the displacement [14] and in the Hausdorff metric of the crack [10]. Alternatively, it is possible to employ energetic evolutions, see e.g. [9,13,17], or the $\varepsilon$-slide approach of [22].

The phase field approach offers instead a regularized framework in which general crack paths are represented by a (phase field) function in such way that the energy is of class $C^{1}$ in a Sobolev space, usually $H^{1}$. The regularity of the energy turns out to be useful both theoretically, since it allows $B V$ solutions, and numerically, since it allows efficient descent algorithms. Several numerical studies indicate a good agreement with experimental results, see e.g. [5].

In statics a precise link between the two approaches is provided by several $\Gamma$-convergence results $[3,9]$; for quasi-static propagations a link is established in full generality only for energetic solutions [19] while for $B V$ solutions it is proved only is some special cases [4,30,36]; at the moment general results like [34] are not at hand. 


\subsection{Energy}

Let $\Omega$ be an open, bounded Lipschitz set in $\mathbb{R}^{2}$. Let $\partial_{D} \Omega \subset \partial \Omega$ with $\mathcal{H}^{1}\left(\partial_{D} \Omega\right)>0$. For $p>2$ let $g \in$ $W^{1, p}\left(\Omega, \mathbb{R}^{2}\right)$. For $t \in[0, T]$ let the space of admissible displacement be

$$
\mathcal{U}(t)=\left\{u \in H^{1}\left(\Omega, \mathbb{R}^{2}\right): u=\operatorname{tg} \text { in } \partial_{D} \Omega\right\} .
$$

Denoting by $\mathcal{U}$ the space $\mathcal{U}(1)$ we can write $\mathcal{U}(t)=t \mathcal{U}$. For the moment we assume that the phase-field variable $v$ belongs to the space $\mathcal{V}=H^{1}(\Omega)$ and in particular we do not impose the bounds $0 \leq v \leq 1$. For $\varepsilon>0$ and $0<\eta_{\varepsilon}=o(\varepsilon)$ the energy functional is the Ambrosio-Tortorelli energy [3] for linear elasticity:

$$
\int_{\Omega}\left(v^{2}+\eta_{\varepsilon}\right) W(D u) \mathrm{d} x+G_{c} \int_{\Omega}(v-1)^{2} / 4 \varepsilon+\varepsilon|\nabla v|^{2} \mathrm{~d} x,
$$

where $G_{c}$ is the toughness and $W$ is the linear elastic energy density, i.e.

$$
W(D u)=\frac{1}{2} D u: \mathbf{C} D u, \quad \mathbf{C} D u=\left(2 \mu \varepsilon(u)+\lambda \operatorname{tr}(\varepsilon(u)) I, \quad \varepsilon(u)=\frac{1}{2}\left(D u+D u^{T}\right) .\right.
$$

Thanks to the 2-homogeneity of the density $W$, it is possible (and convenient) to consider the evolution for the energy $F_{\varepsilon}:[0, T] \times \mathcal{U} \times \mathcal{V} \rightarrow \mathbb{R}$ given by

$$
F_{\varepsilon}(t, u, v)=t^{2} \int_{\Omega}\left(v^{2}+\eta_{\varepsilon}\right) W(D u) \mathrm{d} x+G_{c} \int_{\Omega}(v-1)^{2} / 4 \varepsilon+\varepsilon|\nabla v|^{2} \mathrm{~d} x .
$$

It is convenient to introduce also a notation for the elastic energy and the dissipation potential, respectively

$$
\begin{aligned}
E_{\varepsilon}(t, u, v) & =t^{2} \int_{\Omega}\left(v^{2}+\eta_{\varepsilon}\right) W(D u) \mathrm{d} x, \\
\mathcal{D}_{\varepsilon}(v) & =G_{C} \int_{\Omega}(v-1)^{2} / 4 \varepsilon+\varepsilon|\nabla v|^{2} \mathrm{~d} x .
\end{aligned}
$$

Since we are concerned with quasi-static evolutions, we can "condense" the energy considering only the displacement at equilibrium, to this end let $u(v)$ be the unique minimizer of $E_{\varepsilon}(t, \cdot, v)$ and denote

$$
\begin{aligned}
& \mathcal{E}_{\varepsilon}(t, v)=E_{\varepsilon}(t, u(v), v)=\min \left\{E_{\varepsilon}(t, u, v): u \in \mathcal{U}\right\}, \\
& \mathcal{F}_{\varepsilon}(t, v)=\mathcal{E}_{\varepsilon}(t, v)+\mathcal{D}_{\varepsilon}(v)=\min \left\{F_{\varepsilon}(t, u, v): u \in \mathcal{U}\right\} .
\end{aligned}
$$

Finally, note that, if $v(t)$ is an absolutely continuous trajectory, the dissipation (rate of dissipated energy) is given by

$$
d_{t} \mathcal{D}_{\varepsilon}(v(t))=d_{v} \mathcal{D}(v(t))[\dot{v}(t)]=2 G_{c} \int_{\Omega}(v(t)-1) \dot{v}(t) / 4 \varepsilon+\varepsilon \nabla v(t) \cdot \nabla \dot{v}(t) \mathrm{d} x .
$$

In particular the dissipation depends on the state $v$ and is linear with respect to $\dot{v}$ (the latter is indeed always the case when there exists a dissipation potential).

In the evolution, the irreversibility of the crack is given by the monotonicity constraint $v\left(t_{2}\right) \leq v\left(t_{1}\right)$ if $t_{2} \geq t_{1}$. Hence, given $v \in \mathcal{V}$ the set of admissible variations is the cone

$$
\Phi=\{v \in \mathcal{V}: v \leq 0\}
$$

In analogy with the un-constrained case let us define the unilateral slope

$$
\left|\partial_{v} \mathcal{F}_{\varepsilon}(t, v)\right|=\max \left\{-\partial_{v} \mathcal{F}_{\varepsilon}(t, v)[\phi]: \phi \in \Phi,\|\phi\| \leq 1\right\} .
$$


Note that the functions $\phi$ appearing in the definition of the slope belong to the cone $\Phi$ of admissible variations, thus in general the slope does not coincide with

$$
\left\|\partial_{v} \mathcal{F}_{\varepsilon}(t, v)\right\|=\max \left\{\partial_{v} \mathcal{F}_{\varepsilon}(t, v)[\phi]:\|\phi\| \leq 1\right\} .
$$

Moreover, since $-\Phi \neq \Phi$ it is not possible to write that

$$
\max \left\{-\partial_{v} \mathcal{F}_{\varepsilon}(t, v)[\phi]: \phi \in \Phi,\|\phi\| \leq 1\right\}=\max \left\{\partial_{v} \mathcal{F}_{\varepsilon}(t, v)[\phi]: \phi \in \Phi,\|\phi\| \leq 1\right\} .
$$

Since $\phi=0$ is an admissible variation we always have $\left|\partial_{v} \mathcal{F}_{\varepsilon}(t, v)\right| \geq 0$. Note also that $\Phi$ is weakly closed and that it is not restrictive to choose $\|\phi\|=1$ in the definition of the slope.

The energy $\mathcal{F}_{\varepsilon}$ and its derivatives have been studied in detail in the recent work [21], the interested reader will find there the proof of the following Lemma which provides the properties, corresponding to (2.3)-(2.5), employed in the existence result.

Lemma 8.1. The functional $\mathcal{F}_{\varepsilon}:[0, T] \times \mathcal{V} \rightarrow[0,+\infty)$ is of class $C^{1}$ with

$$
\begin{gathered}
\partial_{t} \mathcal{F}_{\varepsilon}(t, v)=2 t \int_{\Omega}\left(v^{2}+\eta_{\varepsilon}\right) D u(v): \mathbf{C} D g \mathrm{~d} x \\
\partial_{v} \mathcal{F}_{\varepsilon}(t, v)[\phi]=2 t^{2} \int_{\Omega} v \phi W(D u(v)) \mathrm{d} x+2 G_{c} \int_{\Omega}(v-1) \phi / 4 \varepsilon+\varepsilon \nabla v \cdot \nabla \phi \mathrm{d} x .
\end{gathered}
$$

There exists a constant $C$ such that

$$
\left|\partial_{v} \mathcal{F}_{\varepsilon}(t, v)[\phi]-\partial_{v} \mathcal{F}_{\varepsilon}(t, w)[\phi]\right|+\left|\partial_{t} \mathcal{F}_{\varepsilon}(t, v)-\partial_{t} \mathcal{F}_{\varepsilon}(t, w)\right| \leq C\|v-w\|
$$

for every $t \in[0, T]$ and for every $\phi \in \Phi$ with $\|\phi\| \leq 1$. Moreover,

$$
\begin{aligned}
\mathcal{F}_{\varepsilon}(t, v) & \leq \liminf _{m} \mathcal{F}_{\varepsilon}\left(t_{m}, v_{m}\right) \quad \text { for } t_{m} \rightarrow t \text { and } v_{m} \rightarrow v \\
\left|\partial_{v} \mathcal{F}_{\varepsilon}(t, v)\right| & \leq \liminf _{m}\left|\partial_{v} \mathcal{F}_{\varepsilon}\left(t_{m}, v_{m}\right)\right| \quad \text { for } t_{m} \rightarrow t \text { and } v_{m} \rightarrow v \\
\partial_{t} \mathcal{F}_{\varepsilon}(t, v) & =\lim _{m} \partial_{t} \mathcal{F}_{\varepsilon}\left(t_{m}, v_{m}\right) \quad \text { for } t_{m} \rightarrow t \text { and } v_{m} \rightarrow v .
\end{aligned}
$$

Proof. For (8.1)-(8.2) see Lemma 2.7 in [21]. For (8.4)-(8.6) see Corollary 2.9 in [21]. For (8.3) see respectively (2.28) and (2.36) in [21] while the continuity of $\partial_{v} \mathcal{D}_{\varepsilon}$ is standard.

\subsection{Evolution in the $\mathbf{H}^{1}$-norm}

We use the implicit scheme of Section 4 . Let $\Delta \tau_{n} \rightarrow 0^{+}$. Given the initial conditions $t_{n, 0}=0$ and $v_{n, 0}=v_{0} \leq 1$ and known $t_{n, k}<T$ and $v_{n, k}$, the updates $v_{n, k+1}$ and $t_{n, k+1}$ are defined by

$$
\left\{\begin{array}{l}
v_{n, k+1} \in \operatorname{argmin}\left\{\mathcal{F}_{\varepsilon}\left(t_{n, k}, v\right): v \in \mathcal{V}, v \leq v_{n, k},\left\|v-v_{n, k}\right\| \leq \Delta \tau_{n}\right\}, \\
t_{n, k+1}=t_{n, k}+\left(\Delta \tau_{n}-\left\|v_{n, k+1}-v_{n, k}\right\|\right) .
\end{array}\right.
$$

As in Section 4 for $\bar{k}_{n}=\sup \left\{k: t_{n, k}<T\right\}$ let $\mathcal{T}_{n}=\bar{k}_{n} \Delta \tau_{n}$ and $\tau_{n, k}=k \Delta \tau_{n}$ for $0 \leq k \leq \bar{k}_{n}$. Next, we define the affine interpolations $\left(t_{n}, v_{n}\right):\left[0, \mathcal{T}_{n}\right) \rightarrow[0, T] \times \mathcal{V}$ which are Lipschitz continuous and satisfy $t_{n}^{\prime} \geq 0$ and $t_{n}^{\prime}+\left\|v_{n}^{\prime}\right\|=1$ a.e. in $\left(0, \mathcal{T}_{n}\right)$. Let us see the discrete equilibrium and energy inequality.

Proposition 8.2. If $t_{n, k+1}>t_{n, k}$ then $v_{n, k+1}$ satisfies the equilibrium condition

$$
\left|\partial_{v} \mathcal{F}_{\varepsilon}\left(t_{n, k}, v_{n, k+1}\right)\right|=0 .
$$


Proof. Since $\left\|v_{n, k+1}-v_{n, k}\right\|<\Delta \tau_{n}$, the minimality of $v_{n, k+1}$ implies that

$$
\lim _{h \rightarrow 0^{+}} \frac{\mathcal{F}_{\varepsilon}\left(t, v_{n, k+1}+h \phi\right)-\mathcal{F}_{\varepsilon}\left(t, v_{n, k+1}\right)}{h}=0,
$$

which gives $\partial_{v} \mathcal{F}_{\varepsilon}\left(t_{n, k}, v_{n, k+1}\right)[\phi]=0$ for every $\phi \in \Phi$.

Proposition 8.3. The following incremental energy estimate holds

$$
\begin{aligned}
\mathcal{F}_{\varepsilon}\left(t_{n, k+1}, v_{n, k+1}\right) \leq & \mathcal{F}_{\varepsilon}\left(t_{n, k}, v_{n, k}\right)-\int_{\tau_{n, k}}^{\tau_{n, k+1}}\left|\partial_{v} \mathcal{F}_{\varepsilon}\left(t_{n, k}, v_{n}(\tau)\right)\right| \mathrm{d} \tau \\
& +\int_{\tau_{n, k}}^{\tau_{n, k+1}} \partial_{t} \mathcal{F}_{\varepsilon}\left(t_{n}(\tau), v_{n}(\tau)\right) t_{n}^{\prime}(\tau) \mathrm{d} \tau+3 C\left\|v_{n, k}-v_{n, k+1}\right\| \Delta \tau_{n},
\end{aligned}
$$

where $C$ is the Lipschitz constant appearing in (8.3).

Proof. We can argue exactly as in the proof of Theorem 4.2, replacing the norm $\left\|\partial \mathcal{F}_{\varepsilon}(t, v)\right\|$ with the slope $\left|\partial_{v} \mathcal{F}_{\varepsilon}(t, v)\right|$ and using (8.3).

Proposition 8.4. Let $\left(t_{n}, v_{n}\right):\left[0, \mathcal{T}_{n}\right) \rightarrow[0, T] \times \mathcal{V}$ be given as above. Let $0<\mathcal{T}<\liminf _{n} \mathcal{T}_{n}$. There exists a subsequence (not relabelled) such that $t_{n} \stackrel{*}{\rightarrow} t$ in $W^{1, \infty}(0, \mathcal{T})$ and $v_{n} \stackrel{*}{\rightarrow} v$ in $W^{1, \infty}(0, \mathcal{T} ; \mathcal{V})$. In particular $t_{n}\left(\tau_{n}\right) \rightarrow t(\tau)$ and $v_{n}\left(\tau_{n}\right) \rightarrow v(\tau)$ in $\mathcal{V}$ if $\tau_{n} \rightarrow \tau$. Moreover $0 \leq t^{\prime} \leq 1, v^{\prime} \leq 0$ and $\left\|v^{\prime}\right\| \leq 1$ a.e. in $(0, \mathcal{T})$.

Proof. It is sufficient to follow the proof of Proposition 4.3.

Theorem 8.5. There exists (a parametrization of) an evolution $(t, v):[0, \mathcal{T}) \rightarrow[0, T] \times \mathcal{V}$ such that $(t(0), v(0))=$ $\left(0, v_{0}\right), 0 \leq t^{\prime} \leq 1$ and $\left\|v^{\prime}\right\| \leq 1, v^{\prime} \leq 0$; moreover

$\left(S^{\prime}\right)$ for every $\tau$ with $t^{\prime}(\tau)>0$ it holds

$$
\left|\partial_{v} \mathcal{F}_{\varepsilon}(t(\tau), v(\tau))\right|=0
$$

$\left(E^{\prime}\right)$ for every $\tau$ it holds

$$
\mathcal{F}_{\varepsilon}(t(\tau), v(\tau))=\mathcal{F}_{\varepsilon}\left(0, v_{0}\right)-\int_{0}^{\tau}\left|\partial_{v} \mathcal{F}_{\varepsilon}(t(s), v(s))\right| \mathrm{d} s+\int_{0}^{\tau} \partial_{t} \mathcal{F}_{\varepsilon}(t(s), v(s)) t^{\prime}(s) \mathrm{d} s .
$$

Proof. In order to prove $\left(S^{\prime}\right)$ and $\left(E^{\prime}\right)$ it is sufficient again to follow step by step the proof of Theorem 4.4, replacing $\left\|\partial_{v} \mathcal{F}_{\varepsilon}(t, v)\right\|$ with the slope $\left|\partial_{v} \mathcal{F}_{\varepsilon}(t, v)\right|$ and using (8.5) and (8.6).

As a by-product we get also the convergence of the energies and then the strong convergence of the phase field variable.

Corollary 8.6. $\mathcal{F}_{\varepsilon}\left(t_{n}(\tau), v_{n}(\tau)\right) \rightarrow \mathcal{F}_{\varepsilon}(t(\tau), v(\tau))$ and then $v_{n}(\tau) \rightarrow v(\tau)$ strongly in $H^{1}(\Omega)$

Proof. The convergence of the energy follows from Corollary 4.5. For the strong convergence of the phase field variable it is instead enough to observe that $\mathcal{E}_{\varepsilon}\left(t_{n}(\tau), v_{n}(\tau)\right) \rightarrow \mathcal{E}_{\varepsilon}(t(\tau), v(\tau))$ if $t_{n}(\tau) \rightarrow t(\tau)$ and $v_{n}(\tau) \rightarrow v(\tau)$. Since $\mathcal{F}_{\varepsilon}\left(t_{n}(\tau), v_{n}(\tau)\right) \rightarrow \mathcal{F}_{\varepsilon}(t(\tau), v(\tau))$ it follows that $\mathcal{D}_{\varepsilon}\left(v_{n}(\tau)\right) \rightarrow \mathcal{D}_{\varepsilon}(v(\tau))$ from which the strong convergence of $v_{n}(\tau)$. 


\subsection{Evolution in the $L^{2}$-norm}

In this section we will show the existence of a quasi-static evolution with respect to the $L^{2}$-norm. The first step is the definition of a slope which takes into account the irreversibility constraint:

$$
\left|\nabla_{L^{2}} \mathcal{F}_{\varepsilon}(t, v)\right|=\max \left\{-\partial_{v} \mathcal{F}_{\varepsilon}(t, v)[\phi]: \phi \in \Phi,\|\phi\|_{L^{2}} \leq 1\right\} .
$$

Theorem 8.7. There exists (a parametrization of) an evolution $(t, v):[0, \mathcal{T}) \rightarrow[0, T] \times \mathcal{V}$ such that $(t(0), v(0))=$ $\left(0, v_{0}\right), 0 \leq t^{\prime} \leq 1,\left\|v^{\prime}\right\| \leq 1$ and $v^{\prime} \leq 0, v \geq 0$ and such that

$\left(S^{\prime}\right)$ for every $\tau$ with $t^{\prime}(\tau)>0$ it holds

$$
\left|\nabla_{L^{2}} \mathcal{F}_{\varepsilon}(t(\tau), v(\tau))\right|=0,
$$

$\left(E^{\prime}\right)$ for every $\tau$ it holds

$$
\mathcal{F}_{\varepsilon}(t(\tau), v(\tau))=\mathcal{F}_{\varepsilon}\left(0, v_{0}\right)-\int_{0}^{\tau}\left|\nabla_{L^{2}} \mathcal{F}_{\varepsilon}(t(s), v(s))\right| \mathrm{d} s+\int_{0}^{\tau} \partial_{t} \mathcal{F}_{\varepsilon}(t(s), v(s)) t^{\prime}(s) \mathrm{d} s .
$$

Proof. Thanks to the properties listed in Lemma 8.1 we can employ the Galerkin approach of Theorem 2.5, see Section 7. Let $\mathcal{V}_{h}$ be a monotone sequence of finite dimensional subspaces of $\mathcal{V}$ with $\cup_{h} \mathcal{V}_{h}$ dense in $\mathcal{V}$ and let $\mathcal{F}_{\varepsilon, h}$ be the restrictions of $\mathcal{F}_{\varepsilon}$ to $\mathcal{V}_{h}$. For $v \in \mathcal{V}_{h}$ let us introduce the slope

$$
\left|\nabla_{L^{2}} \mathcal{F}_{\varepsilon, h}(t, v)\right|=\max \left\{-\partial_{v} \mathcal{F}_{\varepsilon, h}(t, v)[\phi]: \phi \in \Phi \cap \mathcal{V}_{h},\|\phi\|_{L^{2}} \leq 1\right\}
$$

Using (8.3) and the equivalence of norms we get that there exists a constant $C_{h}$ such that

$$
\left|\nabla_{L^{2}} \mathcal{F}_{\varepsilon, h}(t, v)-\nabla_{L^{2}} \mathcal{F}_{\varepsilon, h}(t, w)\right|+\left|\partial_{t} \mathcal{F}_{\varepsilon, h}(t, v)-\partial_{t} \mathcal{F}_{\varepsilon, h}(t, w)\right| \leq C_{h}\|v-w\|_{L^{2}}
$$

for every $t \in[0, T]$ and for every $v, w \in \mathcal{V}_{h}$. Moreover, invoking Lemma 8.1,

$$
\begin{aligned}
\mathcal{F}_{\varepsilon, h}(t, v) & \leq \liminf _{m} \mathcal{F}_{\varepsilon, h}\left(t_{m}, v_{m}\right) \quad \text { for } t_{m} \rightarrow t \text { and } v_{m} \rightarrow v \\
\left|\nabla_{L^{2}} \mathcal{F}_{\varepsilon, h}(t, v)\right| & \leq \liminf _{m}\left|\nabla_{L^{2}} \mathcal{F}_{\varepsilon, h}\left(t_{m}, v_{m}\right)\right| \quad \text { for } t_{m} \rightarrow t \text { and } v_{m} \rightarrow v, \\
\partial_{t} \mathcal{F}_{\varepsilon, h}(t, v) & =\lim _{m} \partial_{t} \mathcal{F}_{\varepsilon, h}\left(t_{m}, v_{m}\right) \quad \text { for } t_{m} \rightarrow t \text { and } v_{m} \rightarrow v
\end{aligned}
$$

As a consequence we can employ the minimizing movement to define a discrete evolution in each space $\mathcal{V}_{h}$ : given $t_{h, n, 0}=0$ and $0 \leq v_{h, n, 0}=v_{h, 0} \leq 1$ and known $t_{h, n, k}<T$ and $v_{h, n, k}$, the incremental problem for $v_{h, n, k+1}$ and $t_{h, n, k+1}$ is given by

$$
\left\{\begin{array}{l}
v_{h, n, k+1} \in \operatorname{argmin}\left\{\mathcal{F}_{\varepsilon, h}\left(t_{h, n, k}, v\right): v \in \mathcal{V}_{h}, v \leq v_{n, k},\left\|v-v_{h, n, k}\right\|_{L^{2}} \leq \Delta \tau_{n}\right\}, \\
t_{h, n, k+1}=t_{h, n, k}+\left(\Delta \tau_{n}-\left\|v_{h, n, k+1}-v_{h, n, k}\right\|_{L^{2}}\right) .
\end{array}\right.
$$

Let us see that $v_{h, n, k+1} \geq 0$ even if this constraint is not explicitly imposed in the incremental problem: by a simple truncation argument for every $v \in \mathcal{V}$ with $v \leq w$ we have $\mathcal{F}_{\varepsilon}(t, v) \geq \mathcal{F}_{\varepsilon}(t, \bar{v})$ and $\|w-v\|_{L^{2}} \geq\|w-\bar{v}\|_{L^{2}}$ for $\bar{v}=\max \{v, 0\}$ (it is interesting to note that in the $H^{1}$-norm in general it is not true that $\|w-v\|_{H^{1}} \geq\|w-\bar{v}\|_{H^{1}}$ ).

Then, using (8.14)-(8.17) and following step by step the previous section we obtain for every $\mathcal{V}_{h}$ a discrete evolution $\left(t_{h}, v_{h}\right):[0, \mathcal{T}) \rightarrow[0, T] \times \mathcal{V}_{h}$ such that $\left(t_{h}(0), v_{h}(0)\right)=\left(0, v_{h, 0}\right), t_{h}^{\prime} \geq 0,\left\|v_{h}^{\prime}\right\| \leq 1, v_{h}^{\prime} \leq 0$ and $v_{h} \geq 0$, moreover

$\left(S_{h}^{\prime}\right)$ for every $\tau$ with $t_{h}^{\prime}(\tau)>0$

$$
\left|\nabla_{L^{2}} \mathcal{F}_{\varepsilon, h}\left(t_{h}(\tau), v_{h}(\tau)\right)\right|=0
$$


$\left(E_{h}^{\prime}\right)$ for every $\tau$

$$
\begin{aligned}
\mathcal{F}_{\varepsilon, h}\left(t_{h}(\tau), v_{h}(\tau)\right)= & \mathcal{F}_{\varepsilon, h}\left(0, v_{h, 0}\right)-\int_{0}^{\tau}\left|\nabla_{L^{2}} \mathcal{F}_{\varepsilon, h}\left(t_{h}(s), v_{h}(s)\right)\right| \mathrm{d} s \\
& +\int_{0}^{\tau} \partial_{t} \mathcal{F}_{\varepsilon, h}\left(t_{h}(s), v_{h}(s)\right) t_{h}^{\prime}(s) \mathrm{d} s .
\end{aligned}
$$

The final step consists in passing to the limit with respect to $h$. To this end we will follow the last part of Section 7. Compactness does not present any particular difficulty and provides (up to subsequences) a limit parametrization $(t, v):[0, \mathcal{T}) \rightarrow[0, T] \times \mathcal{V}$ such that $0 \leq t^{\prime} \leq 1, v^{\prime} \leq 0$ and $\left\|v^{\prime}\right\|_{L^{2}} \leq 1$ a.e. in $(0, \mathcal{T})$. It is important to remark that (up to subsequences) $v_{h}(\tau) \rightarrow v(\tau)$ weakly in $H^{1}$ and thus strongly in $L^{2}$. As a consequence the limit evolution will still satisfy also the constraint $0 \leq v(\tau) \leq v_{0}$. Next, to pass to the limit in $\left(S_{h}^{\prime}\right)$ it is enough to check that

$$
\left|\nabla_{L^{2}} \mathcal{F}_{\varepsilon}(t(\tau), v(\tau))\right| \leq \liminf _{h}\left|\nabla_{L^{2}} \mathcal{F}_{\varepsilon, h}\left(t_{h}(\tau), v_{h}(\tau)\right)\right|
$$

Once again, it is enough to follow Section 7.

To conclude let us spend few more words on some results closely related to ours. In [19] it is employed an energetic evolution, i.e. satisfying the $(\mathrm{S})$ and $(\mathrm{E})$ condition, in which the incremental problem is based on global minimization of the phase field energy. In this setting it is possible to show not only the existence of a quasi-static evolution but also the convergence as $\varepsilon \rightarrow 0$ of the phase field evolutions, say $\left(u_{\varepsilon}, v_{\varepsilon}\right)$, to a sharp crack evolution, say $(u, K)$, where $K$ is the crack set. In the abstract context of energetic evolutions, this is an example of the general framework developed in [29]. Comparing with [19] and with [34], it would be of great interest to know if the $B V$ evolutions, say $\left(\tau_{\varepsilon}, u_{\varepsilon}, v_{\varepsilon}\right)$, of Theorems 8.5 and 8.7 converge as $\varepsilon \rightarrow 0$ to a $B V$ evolution, say $(\tau, u, K)$. In its generality this problem is quite hard since it requires at least a notion of slope for the sharp crack problem. However, a couple of positive answers have been obtained recently in [30,36] under some suitable hypotheses which in turn guarantee that the $L^{2}$ distance of the phase field variable is equivalent (up to a multiplicative constant) to the lenght along a known crack path; in the limit as $\varepsilon \rightarrow 0$ it is found an evolution in the sense of $[20,31]$.

In [12] it is used instead a sharp crack approach, in $S B V$, and it is studied a minimizing movement with respect to the $L^{2}$ distance of the displacement. Note that in our approach it is employed instead the $L^{2}$ distance of the phase-field variable; technically the difference is important because the $L^{2}$ distance (of the displacement) in general is not equivalent to the Hausdorff distance of the crack [14]. The evolution of [12] is naturally associated with the $L^{2}$ gradient flow of the energy, which has been recently studied in [4] in the context of the phase field approach.

\section{REFERENCES}

[1] L. Ambrosio, Minimizing movements. Rend. Accad. Naz. Sci. XL Mem. Mat. Appl. 19 (1995) 191-246.

[2] L. Ambrosio, N. Gigli and G. Savaré, Gradient flows in metric spaces and in the space of probability measures. Lect. Math. ETH Zürich. Birkhäuser Verlag, Basel (2005).

[3] L. Ambrosio and V.M. Tortorelli, On the approximation of free discontinuity problems. Boll. Un. Mat. Ital. B 6 (1992) 105-123.

[4] J.-F. Babadjian and V. Millot, Unilateral gradient flow of the Ambrosio-Tortorelli functional by minimizing movements (2012).

[5] B. Bourdin, G.A. Francfort and J.-J. Marigo, Numerical experiments in revisited brittle fracture. J. Mech. Phys. Solids 48 (2000) 797-826.

[6] B. Bourdin, G.A. Francfort and J.-J. Marigo, The variational approach to fracture. J. Elasticity 91 (2008) 5-148.

[7] A. Braides, $\Gamma$-convergence for beginners. Oxford University Press, Oxford (2002).

[8] A. Braides, Local Minimization, Variational Evolution and $\Gamma$-convergence, vol. 2094 of Lect. Notes Math. Springer, Berlin (2013).

[9] A. Chambolle, A density result in two-dimensional linearized elasticity and applications. Arch. Ration. Mech. Anal. 167 (2003) $211-233$. 
[10] A. Chambolle, G.A. Francfort and J.-J. Marigo, Revisiting Energy Release Rates in Brittle Fracture. J. Nonlinear Sci. 20 (2010) 395-424.

[11] G. Dal Maso, An introduction to $\Gamma$-convergence. Birkhäuser, Boston (1993).

[12] G. Dal Maso and R. Toader, A model for the quasi-static growth of brittle fractures based on local minimization. Math. Models Methods Appl. Sci. 12 (2002) 1773-1799.

[13] G. Dal Maso and R. Toader, A model for the quasi-static growth of brittle fractures: existence and approximation results. Arch. Ration. Mech. Anal. 162 (2002) 101-135.

[14] G. Dal Maso and R. Toader, On a notion of unilateral slope for the Mumford-Shah functional. NoDEA Nonlin. Differ. Equ. Appl. 13 (2007) 713-734.

[15] E. De Giorgi, New problems on minimizing movements. In Boundary value problems for partial differential equations and applications. RMA Res. Notes Appl. Math. Masson, Paris (1993) 81-98.

[16] M.A. Efendiev and A. Mielke, On the rate-independent limit of systems with dry friction and small viscosity. J. Convex Anal. 13 (2006) 151-167.

[17] G.A. Francfort and C.J. Larsen, Existence and convergence for quasi-static evolution in brittle fracture. Commun. Pure Appl. Math. 56 (2003) 1465-1500.

[18] G.A. Francfort and J.-J. Marigo, Revisiting brittle fracture as an energy minimization problem. J. Mech. Phys. Solids 46 (1998) 1319-1342.

[19] A. Giacomini, Ambrosio-Tortorelli approximation of quasi-static evolution of brittle fractures. Calc. Var. Partial Differ. Equ. 22 (2005) 129-172.

[20] D. Knees, A. Mielke and C. Zanini, On the inviscid limit of a model for crack propagation. Math. Models Methods Appl. Sci. 18 (2008) 1529-1569.

[21] D. Knees, R. Rossi and C. Zanini, A vanishing viscosity approach to a rate-independent damage model (2013).

[22] C.J. Larsen, Epsilon-stable quasi-static brittle fracture evolution. Comm. Pure Appl. Math. 63 (2010) 630-654.

[23] C.J. Larsen, C. Ortner and E. Süli, Existence of solutions to a regularized model of dynamic fracture. Math. Models Methods Appl. Sci. 20 (2010) 1021-1048.

[24] A. Mielke, Evolution of rate-independent systems, volume Evolutionary equations. Handb. Differ. Equ. Elsevier, Amsterdam (2005) 461-559.

[25] A. Mielke, Differential, energetic, and metric formulations for rate-independent processes. In Nonlinear PDE's and applications, vol. 2028 of Lecture Notes in Math. Springer, Heidelberg (2011) 87-170.

[26] A. Mielke, R. Rossi and G. Savaré, Modeling solutions with jumps for rate-independent systems on metric spaces. Discrete Contin. Dyn. Syst. 25 (2009) 585-615.

[27] A. Mielke, R. Rossi, and G. Savaré, BV solutions and viscosity approximations of rate-independent systems. ESAIM: COCV 18 (2012) 36-80.

[28] A. Mielke, R. Rossi and G. Savaré, Variational convergence of gradient flows and rate-independent evolutions in metric spaces. Milan J. Math. 80 (2012) 381-410.

[29] A. Mielke, T. Roubíček and U. Stefanelli, $\Gamma$-limits and relaxations for rate-independent evolutionary problems. Calc. Var. Partial Differ. Equ. 31 (2008) 387-416.

[30] M. Negri, From phase-field to sharp cracks: convergence of quasi-static evolutions in a special setting. Appl. Math. Lett. 26 (2013) 219-224.

[31] M. Negri and C. Ortner, Quasi-static propagation of brittle fracture by Griffith's criterion. Math. Models Methods Appl. Sci. 18 (2008) 1895-1925.

[32] C. Ortner, Gradient flows as a selection procedure for equilibria of nonconvex energies. SIAM J. Math. Anal. 38 (2006) 1214-1234.

[33] R. Rossi and G. Savaré, Gradient flows of non convex functionals in Hilbert spaces and applications. ESAIM: COCV 12 (2006) 564-614.

[34] E. Sandier and S. Serfaty, Gamma-convergence of gradient flows with applications to Ginzburg-Landau. Comm. Pure Appl. Math. 57 (2004) 1627-1672.

[35] S. Serfaty, Gamma-convergence of gradient flows on Hilbert and metric spaces and applications. Discrete Contin. Dyn. Syst. 31 (2011) 1427-1451.

[36] P. Sicsic and J.-J. Marigo, From gradient damage laws to Griffith's theory of crack propagation. J. Elasticity 113 (2013) 55-74.

[37] U. Stefanelli, A variational characterization of rate-independent evolution. Math. Nach. 282 (2009) 1492-1512.

[38] R. Toader and C. Zanini, An artificial viscosity approach to quasi-static crack growth. Boll. Unione Mat. Ital. 2 (2009) 1-35. 